\title{
An impulsive modelling framework of fire occurrence in a size structured model of tree-grass interactions for savanna ecosystems
}

\author{
V. Yatat ${ }^{1,2}$, P. Couteron ${ }^{3}$, J.J. Tewa ${ }^{1,2}$, S. Bowong ${ }^{4,2}$, and Y. Dumont ${ }^{5 *}$ \\ ${ }^{1}$ University of Yaounde I, LIRIMA, GRIMCAPE team, Cameroon \\ ${ }^{2}$ IRD, UMI 209, UMMISCO, IRD France Nord, F-93143, Bondy, France \\ ${ }^{3}$ IRD, Umr AMAP, Montpellier, France \\ ${ }^{4}$ University of Douala, LIRIMA, GRIMCAPE team, Cameroon \\ ${ }^{5}$ CIRAD, Umr AMAP, Montpellier, France
}

October 11, 2018

\begin{abstract}
Fires and rainfall are major mechanisms that regulate woody and grassy biomasses in savanna ecosystems. Conditions of long-lasting coexistence of trees and grasses have been mainly studied using continuous-time modelling of tree-grass competition. In these frameworks, fire is a time-continuous forcing while the relationship between woody plant size and fire-sensitivity is not systematically considered. In this paper, we propose a new mathematical framework to model tree-grass interaction that takes into account both the discrete nature of fire occurrence and size-dependent fire sensitivity (via two classes of woody plants). We carry out a qualitative analysis that highlights ecological thresholds and bifurcations parameters that shape the dynamics of the savanna-like systems within the main ecological zones. Moreover, through a qualitative analysis, we show that the impulsive modelling of fire occurrences leads to more diverse behaviors and a more realistic array of solutions than the analogous time-continuous fire models. Numerical simulations are provided to illustrate the theoretical results and to support a discussion about the bifurcation parameters and future developments.
\end{abstract}

key words: Asymmetric competition - Savanna - Fire - Impulsive differential equation Qualitative analysis - Nonstandard finite difference scheme

\section{Introduction}

Savannas are ecosystems with fairly continuous grass cover and variable woody cover (Maurin et al. (2014) [34]). However, savanna-like ecosystems are diverse and cover extensive

\footnotetext{
*Corresponding author: yves.dumont@cirad.fr
} 
areas throughout the tropics. Explanations found in the literature about the possible longlasting coexistence of woody and grassy vegetation components therefore relate to diverse factors and processes depending on the location and the ecological context (Baudena et al. (2014) [11]). Several studies have pointed towards the role of stable ecological factors e.g. climate, in shaping the tree to grass ratio along large-scale gradients of rainfall or soil fertility (Sankaran et al. (2005) [40], (2008) [39]). Other studies have rather emphasized the reaction of vegetation to recurrent disturbances such as herbivory or fire (Langevelde et al. (2003) [31, D'Odorico et al. (2006) [20], Sankaran et al. (2008) [39], Smit et al. (2010) [41, Favier et al. (2012) 24] and references therein). Those two points of view are not mutuallyexclusive since both environmental control and disturbances may co-occur in a given area and along ecological gradients, although their relative importance generally varies among ecosystems. Bond et al. (2003) [14] proposed the name of climate-dependent for ecosystems which physiognomies are highly dependent on climatic conditions (rainfall, soil moisture) versus disturbance-dependent for ecosystems which dynamics is strongly dependent on fires or herbivores.

Several models using a system of ordinary differential equations (ODES) have been proposed to depict and understand the dynamics of woody and herbaceous components in savanna-like vegetation. A first attempt (Walker et al. (1981) [50]) was orientated towards semiarid fireless savannas and analyzed the effect of herbivory and drought on the balance between woody and herbaceous biomass. This model refers to ecosystems immune to fire due to insufficient annual rainfall and grass production. Indeed, fires in savanna-like ecosystems mostly rely on herbaceous biomass that has dried up during the dry season. As long as rainfall is sufficient, fires impact seedlings and saplings within the flame zone and thus let grasses indirectly inhibit tree establishment.

More recently, several attempts have been made (see Langevelde et al. (2003) 31, Accatino et al. (2010) [3, De Michele et al. (2011) [19], Tchuinte et al. (2014) [45], Yatat et al. (2014) [52]) to model the dynamics of savannas, taking into account fires as continuous events, on the basis of the initial framework of Tilman (1994) [46] that used coupled ODES to model the competitive interactions between two kinds of plants.

However, it is questionable to model fire as a continuous forcing that continuously removes fractions of fire sensitive biomass. Indeed, several months and even years can past between two successive fires, such that fire may be considered as an instantaneous perturbation of the savanna ecosystem. Several recent papers have proposed to model fires as stochastic events while keeping the continuous-time differential equation framework (Baudena et al. (2010) 10, Beckage et al. (2011) [12]) or using time discrete matrix models (Accatino \& De Michele (2013) [2]). But in all those examples, fire characteristics remain mainly a linear function of grass biomass which is not satisfactory. Indeed, it is well known that at low grass biomass there is no fires while above a sufficient grass biomass, fires intensity increases rapidly before reaching a saturation. This particular feature of fires in savanna vegetation cannot be modeled by a linear function. Another drawback of the aforementioned recent stochastic models (Baudena et al. (2010) [10, Beckage et al. (2011) [12]) is that they barely lend themselves to analytical (qualitative) approaches.

In this paper, we therefore present a model that differs from most published models and extends the work of Yatat et al. (2014) [52] by modelling discrete fire occurrences. We consider a tree-grass compartmental model. We set one compartment for grass biomass and two for trees, namely fire-sensitive individuals having most of their buds within the flame 
zone (like seedlings, saplings, shrubs) and non-sensitive mature trees having at least their upperparts above the flame zone. We therefore develop a system of three coupled non-linear impulsive differential equations (IDES), one equation per vegetation compartment, that describes savanna dynamics. In addition, we model fire intensity (and the corresponding impact on sensitive woody plants) as an increasing nonlinear and bounded function of grass biomass. Finally, fire occurrences are modeled as pulse-like perturbations.

In order to assess and illustrate the dynamics of some ecological formations through our mathematical model, we distinguish in our numerical computations three climatic zones having distinct characteristics in terms of biomass production. These biomass production zones, loosely relate to Africa and are indexed by their carrying capacity for grass and woody biomasses and by fires return times. Semi-arid areas have a mean annual rainfall that varies between $300 \mathrm{~mm} . \mathrm{yr}^{-1}$ and $650 \mathrm{~mm} . \mathrm{yr}^{-1}$, and fires, if any have return intervals relatively long, says less than one fire event every ten years. Mesic savannas have a mean annual rainfall comprised between $650 \mathrm{~mm} . \mathrm{yr}^{-1}$ and $1100 \mathrm{~mm} . \mathrm{yr}^{-1}$ and fire return time is in order of four or five years, sometimes less. Finally, we consider a humid tropical area with a mean annual rainfall between $1100 \mathrm{~mm} . \mathrm{yr}^{-1}-1800 \mathrm{~mm} . \mathrm{yr}^{-1}$ and where one can have a fire return time from less than one year to two-three years.

Although impulsive differential equations appear highly relevant to model vegetation dynamics in fire prone savannas, they are also difficult in terms of analytical treatments. This may explain why this framework has still remained scarcely used for modelling the dynamics of fire prone savannas. Our model aims to acknowledge three major phenomena: the periodic occurrence of fire events, the fire-mediated, non-linear negative feedback of grasses onto sensitive trees and the negative and/or positive feed-back, depending on location, of insensitive trees on grasses. We therefore explicitly model the occurrence of fires in savanna ecosystems and the asymmetric nature of tree-grass competitive interactions in savannas.

The full impulse fire model of asymmetric tree-grass competition (IFAC) is formulated in Section 2. In Section 3 we reach qualitative analytical results for IFAC through which we highlight some meaningful ecological thresholds that summarize savanna dynamics under impulsive fires. We present a nonstandard numerical scheme for the IFAC model in Section 4 together with the IFAC parameters ranges. In section 5 we present numerical simulations done in the three ecological biomass production areas of the African continent. Moreover, Section 5 also deals with the discussion of our results.

\section{The impulse fire model of asymmetric tree-grass com- petition (IFAC) formulation}

As we have mentioned before, we consider vegetation as composed of three classes, i.e. the class of sensitive tree biomass $\left(T_{S}\right)$, the class of non-sensitive tree biomass $\left(T_{N S}\right)$ and the class of grass biomass $(G)$. We model the fire intensity by a non-linear increasing function of grass biomass $w(G)$. To built up our model, we consider the following assumptions where (A4), (A5), (A6) and (A7) are already described in Yatat et al. (2014) [52].

(A1) A carrying capacity $K_{T}$ for tree biomass (in tons per hectare, $t . h a^{-1}$ ).

(A2) A carrying capacity $K_{G}$ for grass biomass (in tons per hectare, $t . h a^{-1}$ ). 
(A3) Fire events occur periodically, i.e. every $\tau$-time, where $\tau=\frac{1}{f}$ and $f$ denotes the fire frequency.

(A4) Fire only impacts grass and sensitive Tree and, fire intensity is an increasing function of the grass biomass

(A5) The Grass biomass has a direct, depressing effect on the Sensitive Trees.

(A6) Non Sensitive Trees have a depressive or facilitation effect on grass biomass by shading.

(A7) Sensitive tree biomass moves to non-sensitive tree biomass after an averaged time $\frac{1}{\omega_{S}}$ (in years).

The following diagram summarize the relationship between the three compartments

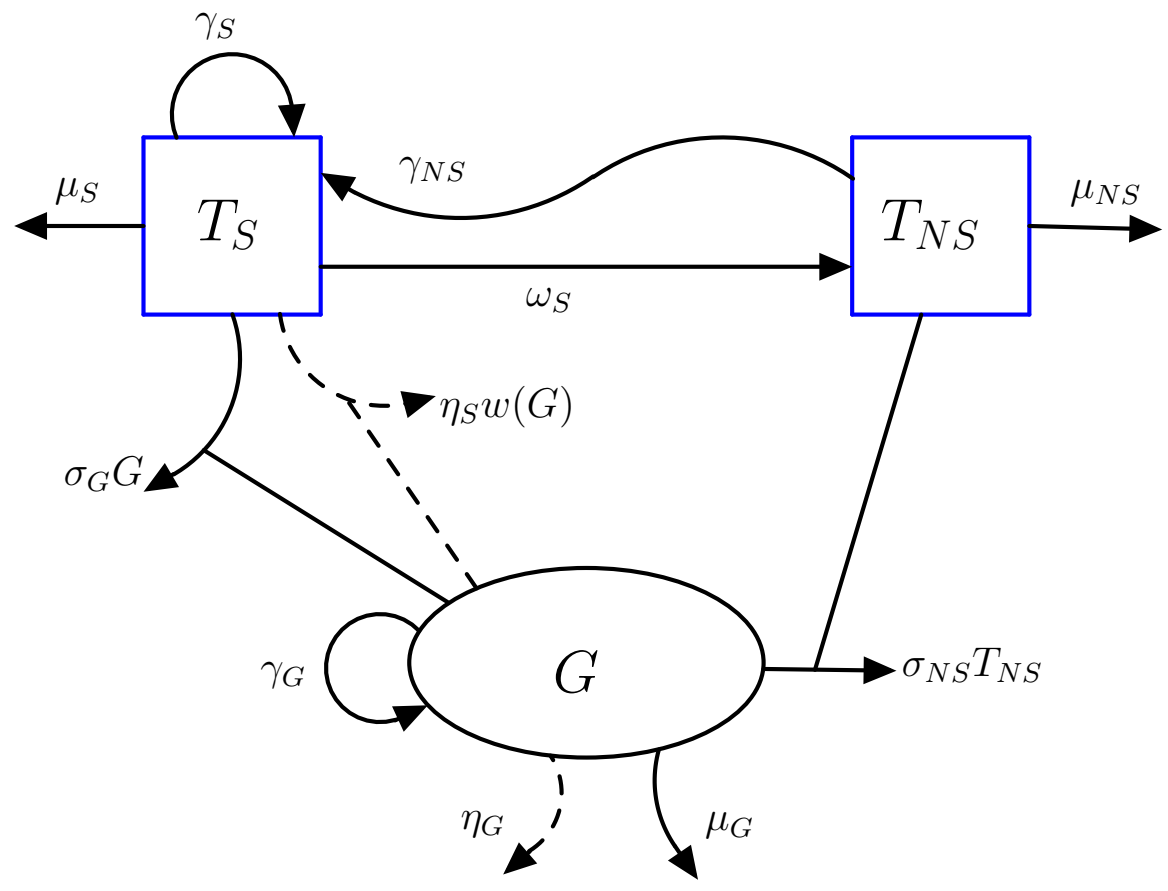

Figure 1: Compartmental diagram of the size structured tree-grass interaction model in impulse fires-prone savanna.

The following parameters are considered throughout the paper:

- Sensitive tree biomass is made up from non sensitive tree biomass (i.e. seed production and germination) with the rate $\gamma_{N S}\left(i n y^{-1}\right)$ and from existing sensitive tree biomass (i.e. intrinsic growth) with the rate $\gamma_{S}\left(\right.$ in $\left.y r^{-1}\right)$.

- Grass biomass is made up from existing grass biomass with the intrinsic growth rate $\gamma_{G}\left(\right.$ in $\left.y r^{-1}\right)$.

- $\mu_{S}\left(\right.$ in $\left.y r^{-1}\right)$ is an additional death rate of sensitive tree biomass due to external disturbances such as human activities and herbivory.

- $\mu_{N S}\left(i n y^{-1}\right)$ is the natural death rate of non sensitive tree biomass. 
- $f$ is the fire frequency (in $y r^{-1}$ ).

- $\mu_{G}\left(\right.$ in $\left.y^{-1}\right)$ is an additional death rate of grass biomass due to factors including human activities and herbivory.

- $\frac{1}{\omega_{S}}$ (in $\left.y r\right)$ is the average time that a sensitive tree takes to become non sensitive to fire.

- $\sigma_{G}$ expresses the asymmetric competition exerted by grasses on sensitive trees (shading and competition for nutrients, in $\left.h a \cdot t^{-1} \cdot y r^{-1}\right)$.

- $\sigma_{N S}$ expresses the asymmetric interaction (competition and/or facilitation) of non sensitive trees on grasses (shading and competition for nutrients, in ha.t $t^{-1} \cdot \mathrm{rr}^{-1}$ ).

- $\eta_{S}$ is the proportion of sensitive tree biomass that is consumed by fire.

- $\eta_{G}$ is the proportion of grass biomass that is consumed by fire.

Based on these ecological premises, and taking into account the effect of fire as pulse phenomena, we propose a model for the savanna vegetation dynamics which is and extension of the model formulated in Yatat et al. (2014) [52].

The IFAC is given by

$$
\begin{aligned}
& \left\{\begin{array}{lll}
\frac{d T_{S}}{d t} & =\left(\gamma_{S} T_{S}+\gamma_{N S} T_{N S}\right)\left(1-\frac{T_{S}+T_{N S}}{K_{T}}\right)-T_{S}\left(\mu_{S}+\omega_{S}+\sigma_{G} G\right), & \\
\frac{d T_{N S}}{d t} & =\omega_{S} T_{S}-\mu_{N S} T_{N S}, & t \neq t_{k} \\
\frac{d G}{d t} & =\gamma_{G}\left(1-\frac{G}{K_{G}}\right) G-\left(\sigma_{N S} T_{N S}+\mu_{G}\right) G, &
\end{array}\right. \\
& \left\{\begin{array}{ll}
T_{S}\left(t_{k}^{+}\right) & =\left(1-\eta_{S} w\left(G\left(t_{k}\right)\right)\right) T_{S}\left(t_{k}\right), \\
T_{N S}\left(t_{k}^{+}\right) & =T_{N S}\left(t_{k}\right), \\
G\left(t_{k}^{+}\right) & =\left(1-\eta_{G}\right) G\left(t_{k}\right),
\end{array} \quad t=t_{k} \quad t_{k+1}=t_{k}+\tau\right.
\end{aligned}
$$

with

$$
T_{S}(0)=T_{S_{0}}>0, T_{N S}(0)=T_{N S_{0}} \geq 0, G(0)=G 0>0 .
$$

For this impulsive fire model, the fire intensity function $w$ is a continuous and positive function of grass biomass which is bounded above by unity. As in Tchuinte et al. (2014) [45] and Yatat et al. (2014) [52, one can choose a generic sigmoidal function (see also Staver et al. (2011) [43]). A typical choice could be

$$
w(G)=\frac{G^{\alpha}}{G^{\alpha}+g_{0}^{\alpha}},
$$

where $G_{0}=g_{0}^{\alpha}$ is the value of grass biomass at which fire intensity reaches its half saturation $\left(g_{0}\right.$ in tons per hectare, $\left.t . h a^{-1}\right)$ and $\alpha \in \mathbb{N}^{*}$.

The feasible region for system (11) - (2) is the set $\Omega$ defined by

$$
\Omega=\left\{\left(T_{S} ; T_{N S} ; G\right) \in \mathbb{R}_{+}^{3} \mid 0 \leq T_{S}+T_{N S} \leq K_{T} ; 0 \leq G \leq K_{G}\right\} .
$$




\section{Mathematical Analysis and Ecological Interpretation of Thresholds}

\subsection{Existence of solution}

The right-hand side of system (1)-(2) is locally lipschitz continuous on $\Omega$. Thus, using a classic existence Theorem (Theorem 1.1 P. 3 in Bainov and Simeonov (1995) [8]), system (1)-(2)-(3) has a unique solution on $\Omega$.

\subsection{Trivial and semitrivial solutions}

It is obvious that system (11) - (2) has always a desert equilibrium $E_{0}=(0,0,0)$.

\subsubsection{The positive grassland periodic solution: existence and local stability}

Let us consider the following thresholds:

$$
\mathcal{R}_{G}^{0}=\frac{\gamma_{G}}{\mu_{G}}, \quad \mu_{G}>0,
$$

and

$$
\rho_{G}^{0}= \begin{cases}\left(1-\eta_{G}\right) \exp \left(\mu_{G}\left(\mathcal{R}_{G}^{0}-1\right) \tau\right), & \mu_{G}>0 \\ \left(1-\eta_{G}\right) \exp \left(\gamma_{G} \tau\right), & \mu_{G}=0 .\end{cases}
$$

Assume that $\mathcal{R}_{G}^{0}>1$, we have the following result (see also Dai et al. (2012) [18])

Lemma 1 When $\rho_{G}^{0}>1$, System (11) - (2) has a positive grassland periodic solution $E_{G}=$ $\left(0 ; 0 ; G^{*}(t)\right)$, where

$$
G^{*}(t)=\left\{\begin{array}{cc}
\frac{K_{G}\left(1-\frac{1}{\mathcal{R}_{G}^{0}}\right)\left(\rho_{G}^{0}-1\right)}{\left(\rho_{G}^{0}-1\right)+\eta_{G} e^{-\mu_{G}\left(\mathcal{R}_{G}^{0}-1\right)(t-(n+1) \tau)},} \quad \mu_{G}>0, & n \tau \leq t<(n+1) \tau, \\
\frac{K_{G}\left(\rho_{G}^{0}-1\right)}{\left(\rho_{G}^{0}-1\right)+\eta_{G} e^{-\gamma_{G}(t-(n+1) \tau)},} & \mu_{G}=0 .
\end{array}\right.
$$

Remark 1 (Thresholds interpretation) $-\mathcal{R}_{G}^{0}$ is the average amount of biomass produced per unit of grass biomass during its whole lifespan in absence of fires and depressing effect from non sensitive trees but subject to additional mortality caused by human activities or by herbivory.

- $\rho_{G}^{0}$ embodies the residual amount of grass biomass at any time-period that fires occur, from the grass biomass produced per unit of grass biomass.

Remark 2 An obvious computation leads to:

$$
\begin{array}{lll}
\mathcal{R}_{G}^{0}<1 & \Longleftrightarrow \rho_{G}^{0}<1, & \\
\rho_{G}^{0}>1 & \Longleftrightarrow \eta_{G}<1-\exp \left(-\mu_{G}\left(\mathcal{R}_{G}^{0}-1\right) \tau\right), & \mu_{G}>0, \\
\rho_{G}^{0}>1 & \Longleftrightarrow \eta_{G}<1-\exp \left(-\gamma_{G} \tau\right), & \mu_{G}=0 .
\end{array}
$$


Let us state the following Lemma which will be helpful for the sequel

Lemma 2 When $\rho_{G}^{0}>1$,

$$
G_{i n t}:=\frac{1}{\tau} \int_{n \tau}^{(n+1) \tau} G^{*}(s) d s=\frac{1}{\tau} \frac{K_{G}}{\gamma_{G}} \ln \left(\rho_{G}^{0}\right)=\frac{1}{\tau} \frac{K_{G}}{\gamma_{G}}\left(\ln \left(1-\eta_{G}\right)+\left(\gamma_{G}-\mu_{G}\right) \tau\right)>0 .
$$

Now we turn to look for local stability of that previous positive and periodic grassland solution. For that purpose, we will use the small perturbation technique and the Floquet theory, i.e. we will find conditions under which all the Floquet multipliers of the positive and periodic grassland solution have their absolute value less than unity or equal to unity (D'Onofrio (2002) [21], Chen et al. (2009) [16]).

Let

$$
\begin{aligned}
& \mathcal{R}_{G}^{T}= \frac{\gamma_{S} \mu_{N S}+\omega_{S} \gamma_{N S}}{\mu_{N S}\left(\mu_{S}+\omega_{S}\right)+\mu_{N S} \sigma_{G} G_{i n t}} \\
&= \frac{\gamma_{S} \mu_{N S}+\omega_{S} \gamma_{N S}}{\mu_{N S}\left(\mu_{S}+\omega_{S}\right)+\frac{\mu_{N S} \sigma_{G}}{\tau} \frac{K_{G}}{\gamma_{G}}\left(\ln \left(1-\eta_{G}\right)+\left(\gamma_{G}-\mu_{G}\right) \tau\right)}, \\
& \mathcal{A}=\gamma_{S} \tau\left(1-\frac{1}{\mathcal{R}}\right)
\end{aligned}
$$

where

$$
\mathcal{R}=\frac{\gamma_{S}}{\mu_{S}+\omega_{S}+\mu_{N S}+\sigma_{G} G_{i n t}}
$$

and

$$
\mathcal{B}=\tau^{2} \mu_{N S}\left(\mu_{S}+\omega_{S}+\sigma_{G} G_{\text {int }}\right)\left(1-\mathcal{R}_{G}^{T}\right) .
$$

Moreover, let $\lambda_{1}, \lambda_{2}$ be the roots of

$$
\mathcal{P}(\lambda)=\lambda^{2}-\mathcal{A} \lambda+\mathcal{B}
$$

and

$$
\rho_{T}=\max \left\{\left(1-\eta_{S} w\left(G^{*}(\tau)\right)\right) e^{\lambda_{1}}, e^{\lambda_{2}}\right\} .
$$

The following result holds for System (11) - (2) .

Lemma 3 (Local stability of the grassland periodic solution $E_{G}$ )

Suppose that the grassland periodic solution $\left(E_{G}\right)$ exists i.e.

$\mathcal{R}_{G}^{0}>1$ and $\rho_{G}^{0}>1$.

Moreover,

- if $\mathcal{R}_{G}^{T}<1$ then, $E_{G}$ is locally asymptotically stable,

- if $\left(\mathcal{R}_{G}^{T}>1\right.$ and $\left.\rho_{T}<1\right)$ then, $E_{G}$ is locally asymptotically stable,

- if $\left(\mathcal{R}_{G}^{T}>1\right.$ and $\left.\rho_{T}=1\right)$ then, $E_{G}$ is locally stable.

- if $\left(\mathcal{R}_{G}^{T}>1\right.$ and $\left.\rho_{T}>1\right)$ then, $E_{G}$ is unstable. 
Proof 1 (See Appendix A.)

Remark 3 (Thresholds interpretation) In the sequel, we provide approximative thresholds interpretation in order to favor an intuitive ecological comprehension of our results with respect to these thresholds.

- $\mathcal{R}_{G}^{T}$ is the sum of the average amount of biomass produced by a sensitive/young plant competing with grass between two successive fires, and the average amount of biomass produced by a mature plant multiplied by the proportion of young plants which reach the mature stage.

- $\rho_{T}$ embodies both the residual of the reduction of trees biomass due to periodic fires events and the reduction of sensitive tree biomass due to competition with grass biomass. Moreover, since $\mathcal{R}<\mathcal{R}_{G}^{T}$, after a direct computation one has

$$
\sigma_{G}<\frac{1}{G_{\text {int }}}\left(\gamma_{S}-\left(\mu_{S}+\omega_{S}+\mu_{N S}\right)\right) \Longrightarrow \rho_{T}>1
$$

Therefore, it clearly appears following relation (13) that the grass vs. sensitive tree competition parameter $\sigma_{G}$ is a bifurcation parameter for the IFAC model that embodies the stability/instability of the grassland periodic solution.

\subsubsection{The positive forest equilibrium: existence and local stability}

Let

$$
\mathcal{R}_{T}^{0}=\frac{\gamma_{S} \mu_{N S}+\gamma_{N S} \omega_{S}}{\mu_{N S}\left(\mu_{S}+\omega_{S}\right)}
$$

The following result follows from Proposition 1 in Yatat et al. (2014) [52].

Lemma 4 If $\mathcal{R}_{T}^{0}>1$ then system (11) -(2) has a positive forest equilibrium $E_{T}=\left(\bar{T}_{S} ; \bar{T}_{N S} ; 0\right)$, where

$$
\begin{aligned}
\bar{T}_{S} & =\quad \frac{K_{T} \mu_{N S}}{\mu_{N S}+\omega_{S}}\left(1-\frac{1}{\mathcal{R}_{T}^{0}}\right), \\
\bar{T}_{N S} & =\frac{\omega_{S}}{\mu_{N S}} \bar{T}_{S}=\frac{K_{T} \omega_{S}}{\mu_{N S}+\omega_{S}}\left(1-\frac{1}{\mathcal{R}_{T}^{0}}\right) .
\end{aligned}
$$

As previously we are checking for local stability of the positive forest equilibrium. Let

$$
\begin{aligned}
\mathcal{R}_{T}^{G} & =\frac{\gamma_{G}}{\mu_{G}+\sigma_{N S} \bar{T}_{N S}} \\
\rho_{T}^{G} & =\left(1-\eta_{G}\right) \exp \left(\gamma_{G}\left(1-\frac{1}{\mathcal{R}_{T}^{G}}\right) \tau\right) .
\end{aligned}
$$

Using the same approach as in the proof of Lemma 3, we derive the following result.

Lemma 5 (Local stability of the forest equilibrium $E_{T}$ )

Suppose that the forest equilibrium $\left(E_{T}\right)$ exists, i.e. $\mathcal{R}_{T}^{0}>1$.

Moreover,

- if $\mathcal{R}_{T}^{G} \leq 1$ then, $E_{T}^{G}$ is locally asymptotically stable, 
- if $\left(\mathcal{R}_{T}^{G}>1\right.$ and $\left.\rho_{T}^{G}<1\right)$ then, $E_{T}$ is locally asymptotically stable,

- if $\left(\mathcal{R}_{T}^{G}>1\right.$ and $\left.\rho_{T}^{G}=1\right)$ then, $E_{T}$ is locally stable.

- if $\left(\mathcal{R}_{T}^{G}>1\right.$ and $\left.\rho_{T}^{G}>1\right)$ then, $E_{T}$ is unstable.

Remark 4 (Thresholds interpretation) As we mentioned before, we provide approximative thresholds interpretation in order to favor an intuitive ecological comprehension of our results with respect to these thresholds.

- $\mathcal{R}_{T}^{0}$ is the sum of the average amount of biomass produced by a sensitive/young plant, without fires and competition from grass, and the average amount of biomass produced by a mature plant multiplied by the proportion of young plants which reach the mature stage.

We may note here that this threshold only depends on the parameters ruling the dynamics of the woody biomass.

- $\mathcal{R}_{T}^{G}$ is the average biomass produced by a unit of grass biomass during its whole lifespan free of fires while experiencing competition from non-sensitive trees.

- $\rho_{T}^{G}$ embodies both the residual grass biomass after a fire event and the residual biomass of the depression of grass biomass due to competition from non-sensitive trees.

One should also note that, when $\mathcal{R}_{T}^{G}>1$, one has

$$
\begin{aligned}
\rho_{T}^{G} \leq 1 & \Longleftrightarrow \eta_{G} \geq 1-\frac{1}{\exp \left(\gamma_{G}\left(1-\frac{1}{\mathcal{R}_{T}^{G}}\right) \tau\right)} \\
& \Longleftrightarrow \tau \leq-\frac{\ln \left(1-\eta_{G}\right)}{\gamma_{G}\left(1-\frac{1}{\mathcal{R}_{T}^{G}}\right)} \\
& \Longleftrightarrow \frac{1}{\bar{T}_{N S}}\left(\gamma_{G}-\mu_{G}+\frac{\ln \left(1-\eta_{G}\right)}{\tau}\right) \leq \sigma_{N S} .
\end{aligned}
$$

Therefore we can deduce three major observations

(i) Firstly, if the fires period $\tau$ is small (i.e. the fires frequency $f$ is high) then $1-\frac{1}{\exp \left(\gamma_{G}\left(1-\frac{1}{\mathcal{R}_{T}^{G}}\right) \tau\right)}$ is small and one can have $\rho_{T}^{G} \leq 1$ for small values of

(ii) Secondly, if the fires period $\tau$ is large (i.e. the fires frequency $f$ is small) then

$$
1-\frac{1}{\exp \left(\gamma_{G}\left(1-\frac{1}{\mathcal{R}_{T}^{G}}\right) \tau\right)}=1-\varepsilon
$$

where $0<\varepsilon<<1$. Thus, $\varepsilon$ can be sufficiently small (such that $\rho_{T}^{G} \leq 1$ ), to have a large destruction of grass biomass (more than 99\%). This is ecologically not 
possible (according to the fact that a part of the grass biomass like roots and even the bottom of the tufts, cannot burn). Therefore, in case of large fires period, having $\rho_{T}^{G} \leq 1$ may likely correspond to a decrease of $\mathcal{R}_{T}^{G}$ (i.e. an increase of $\left.\sigma_{N S}\right)$.

(iii) It is easily deduced from relation (17) that the non sensitive tree vs. grass competition/facilitation parameter $\sigma_{N S}$ and the fire return time $\tau$ are bifurcation parameters for the IFAC model that embody stability/instability of the forest equilibrium.

Remark 5 A direct comparison leads to:

1. $\mathcal{R}_{G}^{T}<\mathcal{R}_{T}^{0}$

2. $\mathcal{R}_{G}^{0}<1 \Longrightarrow \mathcal{R}_{T}^{G}<1, \quad \mu_{G}>0$.

\subsubsection{Global stability of trivial equilibrium (desert) and semi-trivial solutions (grassland periodic solution and the forest equilibrium)}

Here, we state a result concerning the global stability of the desert equilibrium, the forest equilibrium and a result concerning the global stability of the grassland periodic solution. Using the thresholds defined in (66), (7) and (14), we have the following

Theorem $1 \bullet$ Case 1: $\mu_{G}>0$.

1. If $\mathcal{R}_{T}^{0}<1$ and $\mathcal{R}_{G}^{0}<1$ then the desert equilibrium $E_{0}$ is globally asymptotically stable $(G A S)$.

2. If $\mathcal{R}_{T}^{0}>1$ and $\mathcal{R}_{G}^{0}<1$ then the forest equilibrium $E_{T}=\left(\bar{T}_{S}, \bar{T}_{N S}, 0\right)$, where $\left(\bar{T}_{S}, \bar{T}_{N S}\right)$ are given in (15), is globally asymptotically stable.

3. If $\mathcal{R}_{T}^{0}<1, \mathcal{R}_{G}^{0}>1$ and $\rho_{G}^{0}<1$ then the desert equilibrium $E_{0}$ is globally asymptotically stable.

4. If $\mathcal{R}_{T}^{0}<1, \mathcal{R}_{G}^{0}>1$ and $\rho_{G}^{0}>1$ then the grassland periodic solution $E_{G}=\left(0,0, G^{*}(t)\right)$, where $G^{*}(t)$ is given by (8), is globally asymptotically stable.

- Case 2: $\mu_{G}=0$.

(i) If $\mathcal{R}_{T}^{0}<1$ and $\rho_{G}^{0}<1$ then the desert equilibrium $E_{0}$ is globally asymptotically stable.

(ii) If $\mathcal{R}_{T}^{0}<1$ and $\rho_{G}^{0}>1$ then the grassland periodic solution $E_{G}=\left(0,0, G^{*}(t)\right)$, where $G^{*}(t)$ is given by (8), is globally asymptotically stable.

(iii) If $\mathcal{R}_{T}^{0}>1$ and $\rho_{G}^{0}<1$ then the forest equilibrium $E_{T}=\left(\bar{T}_{S}, \bar{T}_{N S}, 0\right)$, where $\left(\bar{T}_{S}, \bar{T}_{N S}\right)$ are given in (15), is globally asymptotically stable.

Proof 2 (See Appendix B.) 


\subsection{Existence of a positive and periodic tree-grass solution}

Now, we reach the position to find at least one non-trivial positive and periodic solution of system (11)-(2). We will use the approach developed by Gaines and Mahwin 1977 [27].

Before we give the main result of this section, we recall useful inequalities according to the thresholds defined in (마) and (7)

1. If $\mathcal{R}_{G}^{0}<1$ then $\rho_{G}^{0}<1$.

2. $\rho_{G}^{0}>1 \Longleftrightarrow\left(\gamma_{G}-\mu_{G}\right)+\frac{\ln \left(1-\eta_{G}\right)}{\tau}>0$.

The following result holds for system (11) - (2).

Theorem 2 (Existence of a positive and periodic savanna solution)

- Case 1: $\mu_{G}>0$.

If $\mathcal{R}_{G}^{0}>1$ and $\rho_{G}^{0}>1$ then system (11) - (2) has at least one positive $\tau$-periodic solution.

- Case 2: $\mu_{G}=0$.

If $\rho_{G}^{0}>1$ then system (11) - (2) has at least one positive $\tau$-periodic solution.

Proof 3 (See Appendix C.)

Remark 6 One should note that Theorem 2 provides only sufficient conditions to ensure existence of at least one positive $\tau$-periodic solution of system (1)-(2). In other words, existence of savanna solution relies on sufficient grass biomass production. Thus, from an ecological point of view assumptions of Theorem 2 can also be view as necessary conditions to have savanna solution.

Remark 7 Note also, that the uniqueness of the positive savanna solution is an open problem. In the rest of the paper, we will assume that we only have one positive solution.

As previously we can check the local asymptotic stability of the positive and periodic savanna solution $E_{T G}=\left(\tilde{T}_{S}(t), \tilde{T}_{N S}(t), \tilde{G}(t)\right)$. Defining

$$
\begin{aligned}
& T_{S}(t)=x(t)+\tilde{T}_{S}(t), \\
& T_{N S}(t)=y(t)+\tilde{T}_{N S}(t), \\
& G(t)=z(t)+\tilde{G}(t),
\end{aligned}
$$

where $x(t), y(t)$ and $z(t)$ are small perturbations and satisfy

$$
\left(\begin{array}{l}
x(t) \\
y(t) \\
z(t)
\end{array}\right)=\Phi(t)\left(\begin{array}{l}
x(0) \\
y(0) \\
z(0)
\end{array}\right)
$$

where $\Phi$ is a fundamental matrix and satisfies

$$
\frac{d \Phi(t)}{d t}=\operatorname{DF}\left(\tilde{T}_{S}(t) ; \tilde{T}_{N S}(t) ; \tilde{G}(t)\right) \Phi(t)=\left(\begin{array}{ccc}
a^{(1)} & a^{(2)} & -\sigma_{G} \tilde{T}_{S}(t) \\
\omega_{S} & -\mu_{N S} & 0 \\
0 & -\sigma_{N S} \tilde{G}(t) & a^{(3)}
\end{array}\right) \Phi(t)
$$


with

$$
\begin{aligned}
& a^{(1)}=\gamma_{S}\left(1-\frac{2 \tilde{T}_{S}(t)+\tilde{T}_{N S}(t)}{K_{T}}\right)-\frac{\gamma_{N S}}{K_{T}} \tilde{T}_{N S}(t)-\sigma_{G} \tilde{G}(t)-\mu_{S}-\omega_{S}, \\
& a^{(2)}=\gamma_{N S}\left(1-\frac{\tilde{T}_{S}(t)+2 \tilde{T}_{N S}(t)}{K_{T}}\right)-\frac{\gamma_{S}}{K_{T}} \tilde{T}_{S}(t), \\
& a^{(3)}=\gamma_{G}\left(1-\frac{2 \tilde{G}(t)}{K_{G}}\right)-\sigma_{N S} \tilde{T}_{N S}(t)-\mu_{G}
\end{aligned}
$$

and $\Phi(0)=I d_{\mathbb{R}^{3}}$. Furthermore, the resetting impulsive condition of system (1)-(2) becomes,

$$
\left(\begin{array}{c}
x\left(n \tau^{+}\right) \\
y\left(n \tau^{+}\right) \\
z\left(n \tau^{+}\right)
\end{array}\right)=\left(\begin{array}{ccc}
1-\eta_{S} w(\tilde{G}(\tau)) & 0 & -w^{\prime}(\tilde{G}(\tau)) \tilde{T}_{S}(\tau) \\
0 & 1 & 0 \\
0 & 0 & 1-\eta_{G}
\end{array}\right)\left(\begin{array}{l}
x(n \tau) \\
y(n \tau) \\
z(n \tau)
\end{array}\right) .
$$

A monodromy matrix $\mathbf{M}$ of system (11) - (2) , is:

$$
\mathbf{M}=\left(\begin{array}{ccc}
1-\eta_{S} w(\tilde{G}(\tau)) & 0 & -w^{\prime}(\tilde{G}(\tau)) \tilde{T}_{S}(\tau) \\
0 & 1 & 0 \\
0 & 0 & 1-\eta_{G}
\end{array}\right) \Phi(\tau)
$$

with

$$
\Phi(t)=\exp \left(\int_{0}^{t} D F\left(\tilde{T}_{S}(s) ; \tilde{T}_{N S}(s) ; \tilde{G}(s)\right) d s\right) .
$$

Let defined $\rho_{T G}$ such as

$$
\rho_{T G}=\max (|\lambda|: \lambda \in \operatorname{sp}(\mathbf{M})),
$$

where the matrix $\mathbf{M}$ is defined in (23). Therefore, following the Floquet theorem (D'Onofrio (2002) [21], Chen et al. (2009) [16]) we deduce the following results

Lemma 6 - If $\rho_{T G}<1$ then the positive $\tau$-periodic solution of system (1) - (2) is locally asymptotically stable.

- If $\rho_{T G}=1$ then the positive $\tau$-periodic solution of system (11) - (2) is locally stable.

- If $\rho_{T G}>1$ then the positive $\tau$-periodic solution of system (11) - (21) is unstable.

Unfortunately, expressions (23) and (24) don't allow an explicit computation of the eigenvalues of the monodromy matrix $\mathbf{M}$ and of the real $\rho_{T G}$. Therefore, the stability of the positive $\tau$-periodic solution of system (11) - (2) will be conjectured through numerical computation of the threshold $\rho_{T G}$.

\subsection{Summary Table of the IFAC model qualitative analysis}

Based on the previous studies, we deduce the summary Table of the qualitative analysis of the IFAC model. In Table 3.4, L stands for Locally Asymptotically Stable, NU stands for 
Numerical Asymptotical Stability and the empty cell denotes either the instability/non existence of the corresponding solution or that the result does not depend on the corresponding threshold. For reader's convenience, we recall all thresholds defined previously:

$$
\begin{aligned}
\mathcal{R}_{T}^{0}= & \frac{\gamma_{S} \mu_{N S}+\gamma_{N S} \omega_{S}}{\mu_{N S}\left(\mu_{S}+\omega_{S}\right)}, \\
\mathcal{R}_{G}^{0}= & \frac{\gamma_{G}}{\mu_{G}}, \quad \text { when } \mu_{G}>0 \\
\rho_{G}^{0}= & \left(1-\eta_{G}\right) \exp \left(\mu_{G}\left(\mathcal{R}_{G}^{0}-1\right) \tau\right), \\
\mathcal{R}_{T}^{G}= & \frac{\gamma_{G}}{\mu_{G}+\sigma_{N S} \bar{T}_{N S}}, \quad \text { where } \bar{T}_{N S}=\frac{K_{T} \omega_{S}}{\mu_{N S}+\omega_{S}}\left(1-\frac{1}{\mathcal{R}_{T}^{0}}\right) \\
\rho_{T}^{G}= & \left(1-\eta_{G}\right) \exp \left(\gamma_{G}\left(1-\frac{1}{\mathcal{R}_{T}^{G}}\right) \tau\right), \\
\mathcal{R}_{G}^{T}= & \frac{\gamma_{S} \mu_{N S}+\omega_{S} \gamma_{N S}}{\left.\mu_{N S}\left(\mu_{S}+\omega_{S}\right)+\frac{\mu_{N S} \sigma_{G} K_{G}}{\tau} \frac{K_{G}}{\gamma_{G}}\left(1-\eta_{G}\right)+\left(\gamma_{G}-\mu_{G}\right) \tau\right)}
\end{aligned}
$$

\begin{tabular}{|c|c|c|c|c|c|c|c|c|}
\hline \multicolumn{5}{|c|}{ Thresholds } & \multicolumn{3}{|c|}{ Solutions } & \multirow[b]{2}{*}{ Case } \\
\hline $\mathcal{R}_{T}^{G}$ & $\rho_{G}^{0}$ & $\rho_{T}^{G}$ & $\mathcal{R}_{G}^{T}$ & $\rho_{T}$ & $E_{T}$ & $E_{G}$ & $E_{T G}$ & \\
\hline \multirow[t]{7}{*}{$>1$} & \multirow[t]{6}{*}{$>1$} & \multirow[t]{3}{*}{$>1$} & \multirow[t]{2}{*}{$>1$} & $>1$ & & & $\mathrm{NU}$ & 1 \\
\hline & & & & $\leq 1$ & & $\mathbf{L}$ & $\mathrm{NU}$ & 2 \\
\hline & & & $<1$ & & & $\mathbf{L}$ & $\overline{\mathrm{NU}}$ & 3 \\
\hline & & \multirow[t]{3}{*}{$\leq 1$} & \multirow[t]{2}{*}{$>1$} & $>1$ & $\mathbf{L}$ & & $\mathrm{NU}$ & 4 \\
\hline & & & & $\leq 1$ & $\mathbf{L}$ & $\mathbf{L}$ & $\mathrm{NU}$ & 5 \\
\hline & & & $<1$ & & $\mathbf{L}$ & $\mathbf{L}$ & $\mathrm{NU}$ & 6 \\
\hline & $<1$ & & & & $\mathbf{L}$ & & $\mathrm{NU}$ & 7 \\
\hline \multirow[t]{4}{*}{$<1$} & \multirow[t]{3}{*}{$>1$} & & \multirow[t]{2}{*}{$>1$} & $>1$ & $\mathbf{L}$ & & $\mathrm{NU}$ & 8 \\
\hline & & & & $\leq 1$ & $\mathbf{L}$ & $\mathbf{L}$ & $\mathrm{NU}$ & 9 \\
\hline & & & $<1$ & & $\mathbf{L}$ & $\mathbf{L}$ & $\mathrm{NU}$ & 10 \\
\hline & $<1$ & & & & $\mathbf{L}$ & & $\mathrm{NU}$ & 11 \\
\hline
\end{tabular}

and $\rho_{T}$ is defined in (12).

In Table 3.4, we implicitly assume that $R_{G}^{0}>1$ and $R_{T}^{0}>1$.

Table 1: Summary table of the qualitative analysis of system (11) - (2). NU: LAS for $E_{T G}$ needs to be estimated numerically, using

Remark 8 In Table 3.4, we only summarizes the cases where $\mathcal{R}_{G}^{0}>1$ and $\mathcal{R}_{T}^{0}>1$, which are the interesting cases from the ecological point of view. Moreover, a direct computation 
leads

$$
\begin{aligned}
\rho_{T}^{G} & =\left(1-\eta_{G}\right) \exp \left(\gamma_{G}\left(1-\frac{1}{\mathcal{R}_{T}^{G}}\right) \tau\right) \\
& =\left(1-\eta_{G}\right) \exp \left(\left(\gamma_{G}-\mu_{G}-\sigma_{N S} \bar{T}_{N S}\right) \tau\right) \\
& =\left(1-\eta_{G}\right) \exp \left(\mu_{G}\left(\mathcal{R}_{G}^{0}-1\right) \tau\right) \exp \left(-\sigma_{N S} \bar{T}_{N S} \tau\right) \\
\rho_{T}^{G} & =\rho_{G}^{0} \exp \left(-\tau \sigma_{N S} \bar{T}_{N S}\right) .
\end{aligned}
$$

Therefore, according to 27), since $\mathcal{R}_{T}^{0}>1$ i.e. $\bar{T}_{N S}>0$ then $\rho_{T}^{G}<\rho_{G}^{0}$.

In the sequel, we provide some numerical simulations in order to illustrate our theoretical results. To achieve that goal, first we will provide a suitable nonstandard numerical scheme which will be helpful for the numerical approximation of the IFAC model solutions. It is well know that standard methods (such as Runge-Kutta or Euler methods) can sometimes present spurious behaviors which are not in adequacy with the system properties that they aim to approximate i.e., lead to negative solutions, exhibit numerical instabilities, or even converge to the wrong equilibrium for certain values of the time discretization or the model parameters (interested readers can also see Yatat et al. (2014) [52], Anguelov et al. (2012) [4], (2013) [5], (2014) [6] and Dumont et al. (2010) [22], (2012) [23] for motivations, details and explanations about nonstandard schemes). Secondly, we will focus on three ecological regions of the African continent that contrast in terms of biomass production conditions, namely a semiarid, a mesic and a humid tropical region, to discuss the IFAC outcomes with respect to published modelling results on savanna ecosystems (Baudena et al. (2014) [11, February et al. (2013) [25], Accatino et al. (2010) [3], Mordelet et al. (1995) [36], Moustakas et al. (2013) [37]).

\section{Nonstandard scheme, parameters ranges and ecolog- ical zones of biomass productions}

\subsection{A nonstandard scheme for the IFAC model}

The nonstandard numerical scheme proposed in this section is adapted from the nonstandard scheme proposed for the COFAC model in Yatat et al. (2014) [52].

System (11) is discretized as follows:

$$
\left\{\begin{aligned}
\frac{G^{k+1}-G^{k}}{\phi_{G}(h)}= & \gamma_{G}\left(1-\frac{G^{k+1}}{K_{G}}\right) G^{k}-\sigma_{N S} T_{N S}^{k} G^{k+1}-\mu_{G} G^{k}, \\
\frac{T_{N S}^{k+1}-T_{N S}^{k}}{\phi(h)}= & \omega_{S} T_{S}^{k}-\mu_{N S} T_{N S}^{k+1}, \\
\frac{T_{S}^{k+1}-T_{S}^{k}}{\phi(h)}= & \left(\gamma_{S}-\left(\mu_{S}+\omega_{S}\right)\right) T_{S}^{k}+\gamma_{N S} T_{N S}^{k+1}-\frac{\gamma_{S}}{K_{T}} T_{S}^{k}\left(T_{S}^{k+1}+T_{N S}^{k+1}\right) \\
& -\frac{\gamma_{N S}}{K_{T}} T_{N S}^{k} T_{N S}^{k+1}-\left(\frac{\gamma_{N S}}{K_{T}} T_{N S}^{k}+\sigma_{G} G^{k}\right) T_{S}^{k+1},
\end{aligned}\right.
$$


and the impulsive event (2) is discretized as follows:

$$
\left\{\begin{array}{l}
G_{+}^{k+1}=\left(1-\eta_{G}\right) G^{k+1} \\
T_{N S+}^{k+1}=T_{N S}^{k+1} \\
T_{S+}^{k+1}=\left(1-\eta_{S} w\left(G^{k+1}\right)\right) T_{S}^{k+1}
\end{array}\right.
$$

where the denominator functions $\phi$ and $\phi_{1}$ read as

$$
\phi(h)=\frac{e^{Q h}-1}{Q}, \quad h>0,
$$

with

$$
Q=\max \left(\mu_{N S}, \gamma_{S}-\left(\mu_{S}+\omega_{S}\right)\right) .
$$

Using the fact that $\gamma_{G}-\mu_{G}=\mu_{G}\left(\mathcal{R}_{G}^{0}-1\right)$, we define

$$
\phi_{G}(h)= \begin{cases}\frac{e^{\mu_{G}\left(\mathcal{R}_{G}^{0}-1\right) h}-1}{\mu_{G}\left(\mathcal{R}_{G}^{0}-1\right)}, \quad \mu_{G}>0 . \\ \frac{e^{\gamma_{G} h}}{\gamma_{G}}, \quad \mu_{G}=0, \quad h>0 .\end{cases}
$$

This scheme is positively invariant and is qualitatively stable, which means that it has the same equilibria than system (11)-(2), and the stability/instability properties of the equilibria are preserved, at least locally, whatever the stepsize $h>0,52$.

\subsection{Parameters ranges and ecological zones of biomass produc- tions}

To provide relevant numerical simulations, one need to use ecologically meaningful parameters ranges and values. Thus, after extensive literature review, we found the following parameters ranges: 
Table 2: Parameters values found in literature

\begin{tabular}{|c|c|c|}
\hline Parameters & values & References \\
\hline \multirow[t]{2}{*}{$f(1 / \tau)$} & $0-1$ & Langevelde et al. (2003) 31 \\
\hline & $0-2$ & Accatino et al. (2010) 3] \\
\hline \multirow[t]{2}{*}{$\gamma_{G}$} & $0.4^{(1)}-4.6^{(2)}$ & (1) Penning de Vries (1982) 38] \\
\hline & & (2) Menaut et al. (1979) 35 \\
\hline \multirow{3}{*}{$\begin{array}{c}\gamma_{S}+\gamma_{N S} \\
\mu_{N S}\end{array}$} & $0.456-7.2$ & Breman et al. (1995) [15] \\
\hline & $0.03-0.3$ & Accatino et al. (2010) [3] \\
\hline & 0.4 & Langevelde et al. (2003) 31] \\
\hline$\mu_{S}$ & $0-0.3$ & Langevelde et al. (2003) 31 \\
\hline$\mu_{G}$ & $0-0.6$ & Langevelde et al. (2003) 31 \\
\hline \multirow[t]{3}{*}{$\eta_{G}$} & $0.1^{(a)}-1^{(b)}$ & (a) Van de Vijver (1999) [48] \\
\hline & & (b) Accatino et al. (2010) 3 \\
\hline & $0.2-1$ & Abbadie et al. (2006) [1] \\
\hline \multirow[t]{3}{*}{$\eta_{S}$} & $0.02-0.6$ & Accatino et al. (2010) 3] \\
\hline & 0.66 & Reinterpretation of Gignoux (1994) [28, \\
\hline & & Reinterpretation of Langevelde et al. (2003) 31 \\
\hline$\omega_{S}$ & $0.05-0.2$ & Wakeling et al. (2011) 49] \\
\hline
\end{tabular}

As stated previously, we will focus our numerical simulations on three ecological zones of the African continent

Region 1 is a semiarid area with mean annual rainfall comprised between $300 \mathrm{~mm} . \mathrm{yr}^{-1}$ and 650 $m m . y r^{-1}$ where there is a low biomass production and few fire occurrence (says one fire event every ten years) if any.

Region 2 is a mesic area with a mean annual rainfall that varies between 650 mm.yr ${ }^{-1}$ and 1100 $m m . y r^{-1}$ which is an intermediate biomass production zone and where we can have on average one fire event every four or five years and sometimes less.

Region 3 is a high biomass production zone, in which we can have one or two fire events per year, i.e. a humid tropical area with a mean annual rainfall between $1100 \mathrm{~mm} . \mathrm{yr}^{-1}$ and 1800 mm.yr ${ }^{-1}$.

Our aim is to assess the different outcomes of the IFAC model along with the influence of the variations of $\sigma_{G}, \sigma_{N S}$ (for which there is no direct information in the published literature) and the fire period $\tau$. The parameter ranges in each of the regions are summarized in Table 3 . 
Table 3: Parameters ranges in the three ecological regions

\begin{tabular}{l|c|c|c}
\hline Parameter & Region 1 & Region 2 & Region 3 \\
\hline$\tau(y r)$ & $>5$ & $2-5$ & $0.5-3$ \\
$K_{T}\left(t . h a^{-1}\right)$ & 30 & $80-90$ & $110-120$ \\
$K_{G}\left(t . h a^{-1}\right)$ & $0-5$ & $5-10$ & $10-20$ \\
$\gamma_{G}\left(y r^{-1}\right)$ & $0.4-2$ & $2-3.5$ & $3.5-4.6$ \\
$\gamma_{S}\left(y r^{-1}\right)$ & $0.2-0.8$ & $0.2-1$ & $1.5-2.7$ \\
$\gamma_{N S}\left(y r^{-1}\right)$ & $0.256-1.2$ & $1.2-2.5$ & $2.5-4.5$ \\
$\mu_{N S}\left(y r^{-1}\right)$ & $0.1-0.25$ & $0.07-0.1$ & $0.02-0.07$ \\
\hline
\end{tabular}

In addition, reinterpreting experiments that concern Region 1 and Region 2 and reported in February et al. (2013) [25] we derived $\sigma_{G}$ (in $\left.h a . t^{-1} . y r^{-1}\right)$ : $0.1843-0.9984$ for Region 1 and $\sigma_{G}: 0.2470-1.6287$ for Region 2. Moreover, several studies located under different rainfall compared grass production under and outside a tree crown. A synthesis was proposed by Mordelet \& Le Roux (see Abadie et al. (2006) Page 156 [1]) that emphasized that the relative production (within to outside) is a decreasing function along the rainfall gradient. We re-interpreted the results as to derive reasonable values for $\sigma_{N S}$ in each of the three regions, using the subsequent reasoning.

Assuming that the measurements were made, free of fires, in grass stands having reached equilibrium, and letting $G_{u}$ and $G_{o}$ be the equilibrium values under and outside crown, respectively. We can write according to the model:

$$
\begin{aligned}
G_{u} & =K_{G}\left(1-\frac{\mu_{G}+\sigma_{N S} \tilde{T}}{\gamma_{G}}\right) \\
G_{o} & =K_{G}\left(1-\frac{\mu_{G}}{\gamma_{G}}\right) .
\end{aligned}
$$

The ratio considered by Mordelet \& Le Roux (1995) [36] (see also Abadie et al. (2006) Page 156 [1]) is:

$$
\delta_{G}=\frac{G_{u}}{G_{o}}
$$

i.e. the ratio of grass production under and outside a tree crown. Assuming $\mu_{G}=0$ we can simplify as:

$$
\delta_{G}=1-\frac{\sigma_{N S} \tilde{T}}{\gamma_{G}} \Longleftrightarrow \sigma_{N S}=\frac{\left(1-\delta_{G}\right) \gamma_{G}}{\tilde{T}} .
$$

$\tilde{T}$ is the woody biomass density to be computed at the scale of an isolated, full grown tree (having reached the maximal height considering the local climate) in any of the three regions. We propose to relate $\tilde{T}$ to $K_{T}$ as

$$
\tilde{T}=\frac{\varepsilon \times K_{T}}{S}
$$

where $S$ is the woody cover characterizing the maximal density $K_{T}$ and $\left.\varepsilon \in\right] 0,1[$ is a coefficient expressing that an isolated tree has less influence on grass production that a complete, closed canopy stand corresponding to $K_{T}$. 
We used the value of $\delta_{G}=1.58$ (resp. 1.25 and $0.75,0.75$ ) of Mordelet \& Le Roux (1995) [36] (see also Abadie et al. (2006) Page 156 [1]) that corresponds to Region 1 (semiarid region) (resp. Region 2, Region 3). Note that $\delta_{G}$ values above 1 express a facilitative effect of trees for grass while values below correspond to a depressing effect. Using also the estimated values for $K_{T}$ and $\gamma_{G}$, we deduce the ranges of variation of $\sigma_{N S}$ (in ha.t ${ }^{-1} . y r^{-1}$ ), summarized in Table 4 .

Table 4: Variation range of $\sigma_{N S}$ in Region 1, Region 2 and Region 3 following reinterpretation of Mordelet \& Le Roux results

\begin{tabular}{c|c|c|c|c|c|c|c|c|}
\cline { 2 - 9 } & $\delta_{G}$ & $\gamma_{G}$ & $K_{T}$ & $\varepsilon(\min )$ & $\varepsilon(\max )$ & $S$ & $\sigma_{N S}(\min )$ & $\sigma_{N S}(\max )$ \\
\hline Region 1 & 1.58 & 0.6 & 30 & 0.4 & 0.75 & 1 & -0.029 & -0.0155 \\
\hline \hline Region 2 & 1.25 & 2.8 & 85 & 0.2 & 0.67 & 1 & -0.0412 & -0.0123 \\
\cline { 2 - 6 } & 0.75 & & & & & & 0.0123 & 0.0412 \\
\hline \hline Region 3 & 0.75 & 4.2 & 115 & 0.1 & 0.15 & 1 & 0.0609 & 0.0913 \\
\hline
\end{tabular}

Note that the range of values for $\sigma_{N S}$ is due to a large uncertainty on $\varepsilon$. Moreover as a straightforward consequence of the results of the reference study (Modelet \& Le Roux (1995) [36], Abadie et al. (2006)) $\sigma_{N S}$ is likely to be negative in Region 1 (shading improves grass production in Region 1, which is also in good agreement with results of Moustakas et al. (2013) [37], Belsky et al. (1989) [13], Weltzin \& Coughenour (1990) [51]).

\section{Numerical simulations and discussion}

\subsection{Results for Region 1}

In semiarid areas, the main mechanisms that govern the ecological processes include

(M1) water limitation on tree growth (Baudena et al. (2014) [11]) and on grass biomass standing crop $\left(K_{G}\right)$

(M2) tree - grass competition, which has an especially strong competitive impact on tree seedlings (February et al. (2013) [25])

(M3) unfrequent fire may reduces woody cover and grass cover but grass biomass recover quickly after fire (Baudena et al. (2014) [11]) while low values of grass biomass limit the impact of competition on sensitive tree biomass.

Point (M2) suggests that the sensitive tree vs. grass competition parameter $\sigma_{G}$ has relatively large values while point (M1) along with point (M3) suggest that woody cover is controlled principally by water availability and secondarily by unfrequent fires (Sankaran et al. (2005) [40]). Thus the depressive effect of scattered woody cover on grass biomass mainly results from reduced light availability and root competition for soil water (see Walker et al. (1981) [50]). Nevertheless, somme references (Belsky et al. (1989) [13], Weltzin \& Coughenour (1990) [51]) also emphasized the facilitation role of scattered tree on grass biomass in East African semiarid savannas. Indeed, compared with the open situation, the highest grass production was recorded under acacia and baobab trees (Mordelet \& Menaut (1995) [36], 
Belsky et al. (1989) [13], Weltzin \& Coughenour (1990) [51]), which are known to have a low light interception and only induce a slight limitation to photosynthesis while shading improves the water balance under the canopy (Barbier et al. 2008) 9]. This finding of Mordelet \& Menaut (1995) [36], Belsky et al. (1989) [13], Weltzin \& Coughenour (1990) [51] can also be explained by the soil enrichment by nitrogen fixing species, like acacias trees which results in a yield increase. Therefore, in semiarid areas the main ecological vegetation types that are observed depending on annual rainfall (which only varies tree/grass ratio) are savannas (February et al. (2013) [25], Baudena et al. (2014) [11], Accatino et al. (2010) [3]) and sometimes forest in the sense of low dry forest, and/or thickets (Walker et al. (1981) [50], Couteron \& Kokou (1997) [17]). The outcome of the IFAC model in semiarid areas is also in adequacy with this previous features. Indeed, let us consider the following table of parameters values

Table 5: Array of parameters' values for Region 1

\begin{tabular}{|c|c|c|c|c|c|c|}
\hline$\gamma_{S}$ & $\gamma_{N S}$ & $\gamma_{G}$ & $\mu_{N S}$ & $\omega_{S}$ & $\mu_{S}$ & $\eta_{S}$ \\
\hline 0.3 & 1 & 0.6 & 0.15 & 0.1 & 0.2 & 0.5 \\
\hline
\end{tabular}

For values in Table 5, we compute

\begin{tabular}{|c|c|}
\hline $\mathcal{R}_{T}^{0}$ & $\mathcal{R}_{G}^{0}$ \\
\hline 3.2222 & 2 \\
\hline
\end{tabular}

and we derive figure 2, 
(A) Level curve of the threshold $\rho_{T}^{\text {G }}$ in Region 1

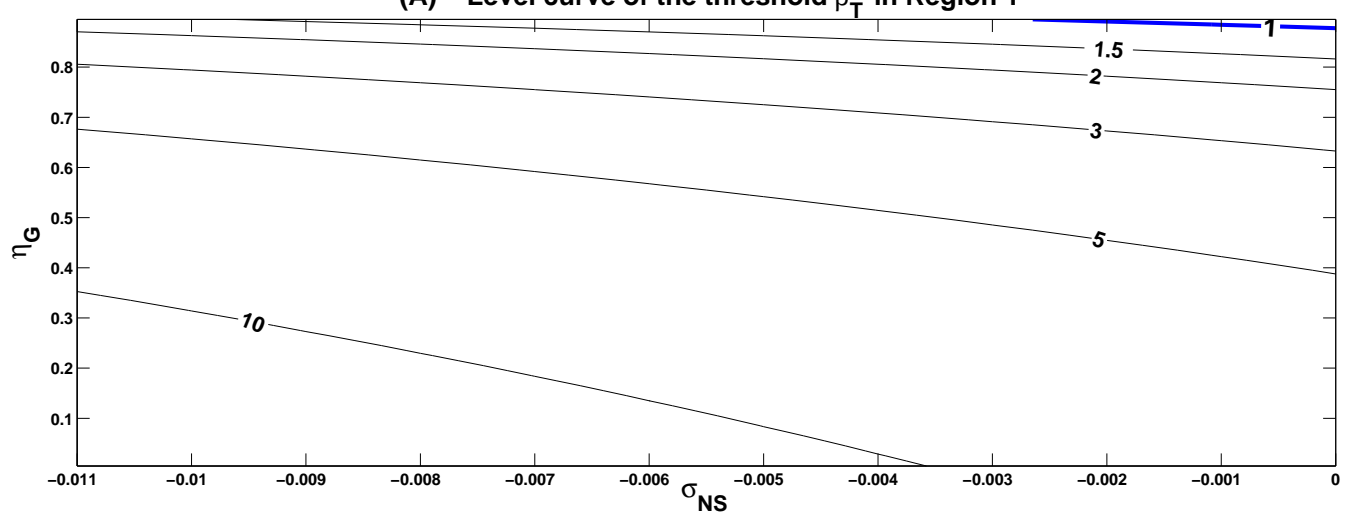

(B) Level curve of the threshold $\rho_{T}^{G}$ in Region 1

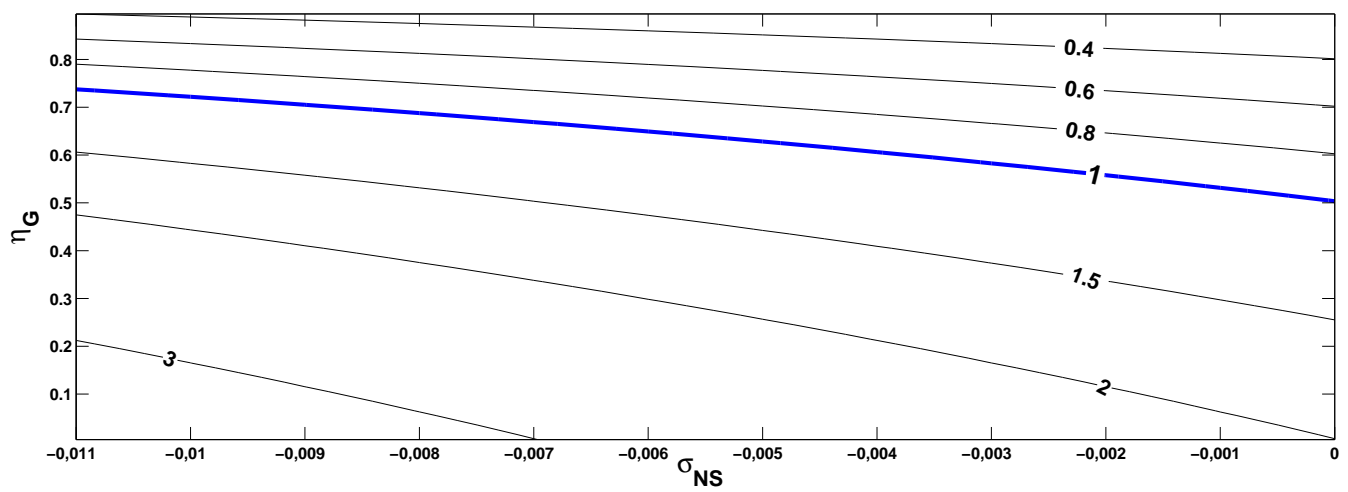

Figure 2: Level curve of the threshold $\rho_{T}^{G}$ illustrating that as $\eta_{G}, \mu_{G}$ and $\sigma_{N S}$ increase, $\rho_{T}^{G}$ decreases and system (11)-(2) is liable to move from a savanna/grassland state to a forest state or to a multistability involving the forest solution. Recall that the forest solution is stable (resp. unstable) whenever $\rho_{T}^{G}$ is lower (resp. greater) than unity. In (A) $\mu_{G}=0.3$, in (B) $\mu_{G}=0.5$.

Recall that according to relation (9),

$$
\rho_{G}^{0}>1 \Longleftrightarrow \eta_{G}< \begin{cases}0.8775 & \text { for } \mu_{G}=0.3 \\ 0.5034 & \text { for } \mu_{G}=0.5\end{cases}
$$

Moreover, together with our data estimation of $\sigma_{G}$ and $\sigma_{N S}$ in Region 1, we found that $\mathcal{R}_{T}^{G}>1$. Setting $\mu_{G}=0.3, \eta_{G}=0.6$, and

- when $\sigma_{G} \in\left[0.92,0.95\left[\right.\right.$, we have $\mathcal{R}_{G}^{T}>1$ and $\rho_{T} \leq 1$

- when $\sigma_{G} \in\left[0.95,0.9984\left[\right.\right.$, we have $\mathcal{R}_{G}^{T} \leq 1$ and $\rho_{T} \leq 1$.

Finally, when $\mu_{G}=0.5$ one gets $\rho_{T}>1$. Therefore, figure 2 together with the previous discussion on $\mathcal{R}_{G}^{T}$ and $\rho_{T}$ illustrates either case 1 to case 6 of Table 3.4. Thus, when external disturbances (such as herbivory) on grass biomass are low and $\sigma_{G}$ has relatively large values, the IFAC model predicts either a stable savanna state, a stable forest state, a stable grassland state or a multistability involving savanna and/or forest and/or grassland (see also case 1, case 2, case 3, case 5 and case 6 of Table 3.4). Furthermore, when external disturbances on grass biomass become more important, the grassland solution becomes unstable and the 
IFAC model predicts either a stable savanna state, a stable forest state or a bistability involving savanna and foret states (see also case 1 and case 4 of Table 3.4). Consequently, one can observe that the non sensitive tree vs. grass interaction parameter $\sigma_{N S}$ and the additional death rate of grass biomass due to external disturbances $\mu_{G}$ are likely to be influential on the IFAC outcomes in Region 1. Nevertheless, with a mean annual rainfall of 300-400 mm there is a wide array of references evidencing the probable bistability of desert (bare soil) and thickets (in the African Sahel) (see Couteron \& Kokou (1997) [17, Lefever et al. (2009) [32, Barbier et al. (2008) [9]) or desert and grass (Namibia) (see Tschinkel (2012) [47, Fernandez-Oto et al. (2014) [26]).

\subsection{Results for Region 2}

In Region 2 which corresponds to a mesic area, the main mechanisms that regulate tree-grass interactions also include mechanisms (M1) and (M2) stated previously for semiarid areas. In addition to (M1) and (M2), in mesic areas, fires are more frequent than in semiarid areas since water availability favor grass biomass production which constitutes the fuel for fires (we denote this new mechanism (M4)). Grass-fire feedback (mechanism M4) together with mechanisms (M1) and (M2), maintain both forest and savanna occurrences in mesic areas (Baudena et al. (2014) [11]). Indeed, grasses benefit from the openness of the landscape after fires, since they recover faster than trees seedlings, thus determining a positive feedback mechanism that enhances savanna presence. The IFAC model also predict a shift from a forest state to a savanna state as $\sigma_{N S}$ decreases and/or when $\sigma_{G}$ increases, which also agree with Baudena et al. (2014) [11] results. Therefore, savanna and forest vegetation types clearly appear as alternatively stable states as found by Staver et al. (2011) [43], Staver and Levin (2012) 44, in our case depending on $\sigma_{G}$ and $\sigma_{N S}$ variation in Region 2.

Let us consider the following table of parameters values

Table 6: Array of parameters' values for Region 2

\begin{tabular}{|c|c|c|c|c|c|c|c|}
\hline$\gamma_{S}$ & $\gamma_{N S}$ & $\gamma_{G}$ & $\mu_{N S}$ & $\omega_{S}$ & $\eta_{S}$ & $\eta_{G}$ & $\mu_{S}$ \\
\hline \hline 0.4 & 2 & 2.8 & 0.08 & 0.1 & 0.5 & 0.6 & 0.1 \\
\hline \hline \multicolumn{10}{|c|}{$K_{T}=85, K_{G}=7, \mu_{G}=0.3$} \\
\hline
\end{tabular}

Using parameters values in Table 6, one has:

\begin{tabular}{|c|c|}
\hline $\mathcal{R}_{T}^{0}$ & $\mathcal{R}_{G}^{0}$ \\
\hline 14.5 & 14 \\
\hline
\end{tabular}

and we also derive figure 3 and figure 4. 


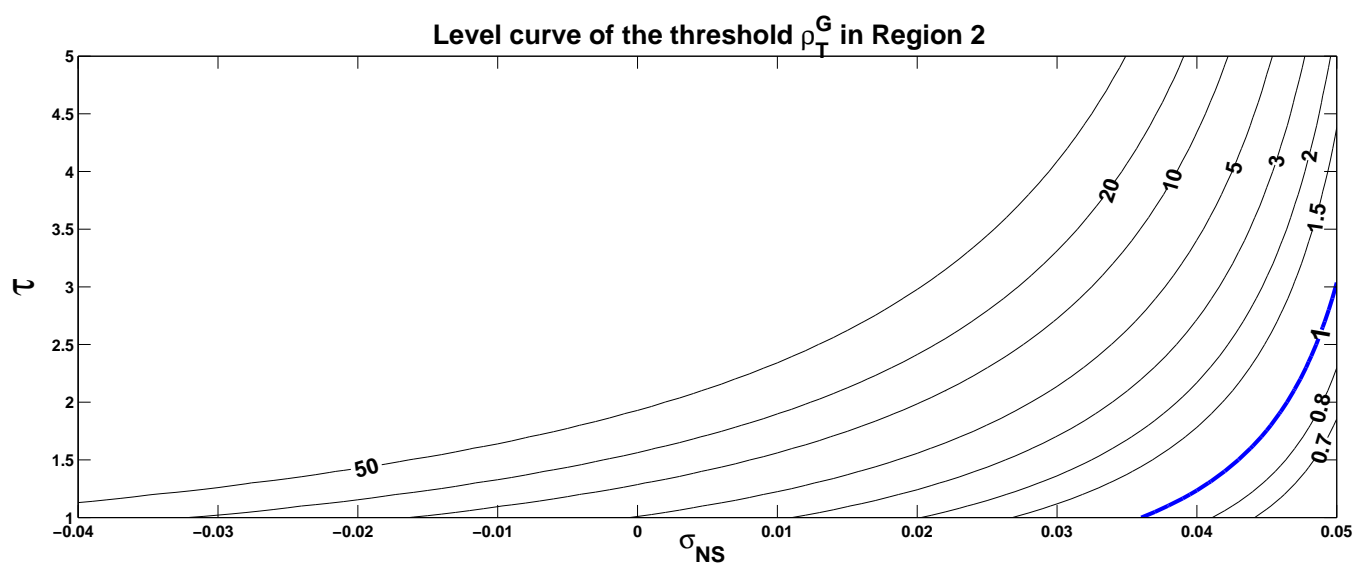

Figure 3: Level curve of the threshold $\rho_{T}^{G}$ illustrating that system (11)-(2) is liable to move from a savanna/grassland state to a forest state or to a multistability involving the forest solution together with $\tau$ and $\sigma_{N S}$ variations. Recall that the forest solution is stable (resp. unstable) whenever $\rho_{T}^{G}$ is lower (resp. greater) than unity.

(A) Level curve of the threshold $\rho_{\mathrm{T}}$ in Region 2

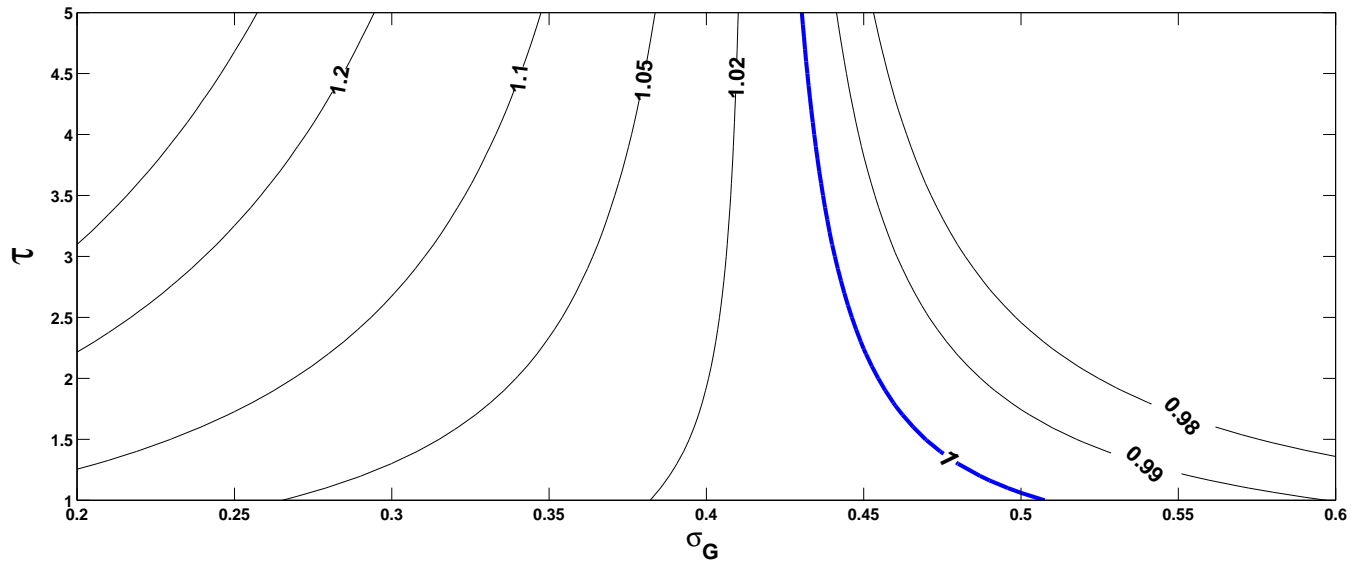

(B) Level curve of the threshold $\rho_{\mathrm{T}}$ in Region 2

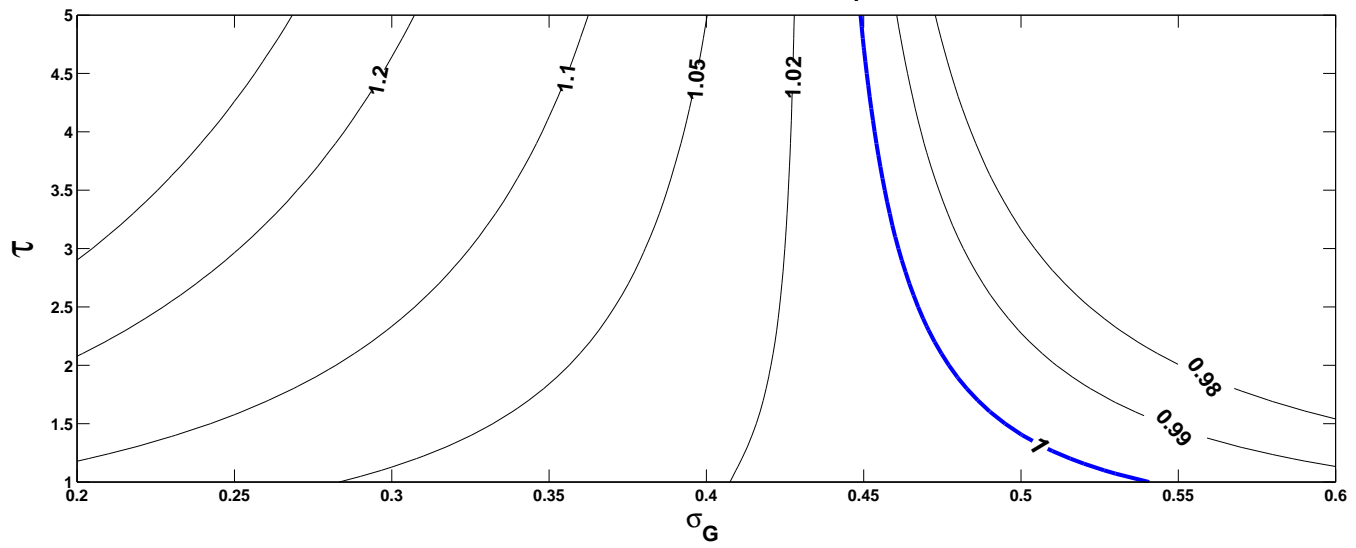

Figure 4: Level curve of the threshold $\rho_{T}$ illustrating that system (11)-(2) is liable to move from a savanna/forest state to a grassland state or to a multistability involving the grassland solution in relation to $\tau$ and $\sigma_{G}$ variations. Recall that the grassland solution is stable (resp. unstable) whenever $\rho_{T}$ is lower (resp. greater) than unity. In (A) $\mu_{G}=0.2$, in (B) $\mu_{G}=0.3$. 
Since for parameters values in Table 6 one has $\mathcal{R}_{G}^{0}>1$ then,

$$
\rho_{G}^{0}>1 \Longleftrightarrow \tau> \begin{cases}0.3524 & \text { for } \mu_{G}=0.2 \\ 0.3665 & \text { for } \mu_{G}=0.3\end{cases}
$$

Moreover, for our estimation of $\sigma_{N S}$ and $\sigma_{G}$ one also has $\mathcal{R}_{T}^{G}>1$ and $\mathcal{R}_{G}^{T}>1$. Therefore, figure 4 and figure 3 illustrate, either case 1, case 2, case 4 or case 5 of Table 3.4 .

In summary, the parameters that are likely to be influential on the IFAC outcomes in Region 2 are the external disturbances on grass biomass parameter $\mu_{G}$, the grass vs. sensitive tree parameter $\sigma_{G}$, the non sensitive tree vs. grass interaction parameter $\sigma_{N S}$. In addition to that previous parameters, one can also mention the fire return time $\tau$. In other words, the previous analysis reveals that in Region 2, in addition of stability of forest, stability of savanna and bistability of forest and savanna as in Region 1, one can observe stability of grassland and also multistabilty situations involving grassland solution with relatively low values of $\sigma_{G}$ in comparison with Region 1 .

\subsection{Results for Region 3}

Region 3 corresponds to humid tropical areas where rainfall availability favors biomass production of both woody and grasses components. The grass-fire feedback possibly leads to stability of either savanna or forest in Region 3 depending on fire return time. Indeed, grass, particularly abundant in these wet areas, becomes an extremely good fuel in the dry season, which promotes fire occurrence and increases fire intensity and impact (Baudena et al. (2014) [11, Higgins et al. (2008) [29]). When the fire return time is large, the trees have the time to grow above the flame zone and to reach canopy closure and then outcompete grasses. Therefore, relatively large return time favor forest state in Region 3 (Staver and Levin (2012) [44]). Moreover, if the fire return time is small then trees don't have the time to reach canopy closure and therefore let grasses which regrow quickly in the open space after fires form either a stable savanna state or a stable grassland state. We illustrate hereafter that these features are also predicted by the IFAC model.

Consider

Table 7: Array of parameters' values for Region 3

\begin{tabular}{|c|c|c|c|c|c|c|c|c|}
\hline$\gamma_{S}$ & $\gamma_{N S}$ & $\gamma_{G}$ & $\mu_{N S}$ & $\omega_{S}$ & $\eta_{S}$ & $\eta_{G}$ & $\mu_{S}$ & $\mu_{G}$ \\
\hline \hline 2 & 3 & 4.2 & 0.06 & 0.1 & 0.5 & 0.6 & 0.1 & 0.2 \\
\hline \hline \multicolumn{10}{|c|}{$K_{T}=115, K_{G}=15$} \\
\hline \hline
\end{tabular}

In this section, we will refer to a particular area, namely the Lamto region in Ivory Coast (see Menaut et al. (1979) [35], Mordelet \& Menaut (1995) [36]). Thanks to Abadie et al. (2006) Page 156 [1] (see also Mordelet \& Menaut (1995) [36]) data report of grass biomass in the canopy and open situations in Lamto and by reinterpreting their results we have derived the range $0.0609 \leq \sigma_{N S} \leq 0.0913$

Using parameters values in Table 7 one has:

\begin{tabular}{|c|c|}
\hline $\mathcal{R}_{T}^{0}$ & $\mathcal{R}_{G}^{0}$ \\
\hline 35 & 21 \\
\hline
\end{tabular}


We also derived the following figure 5 .

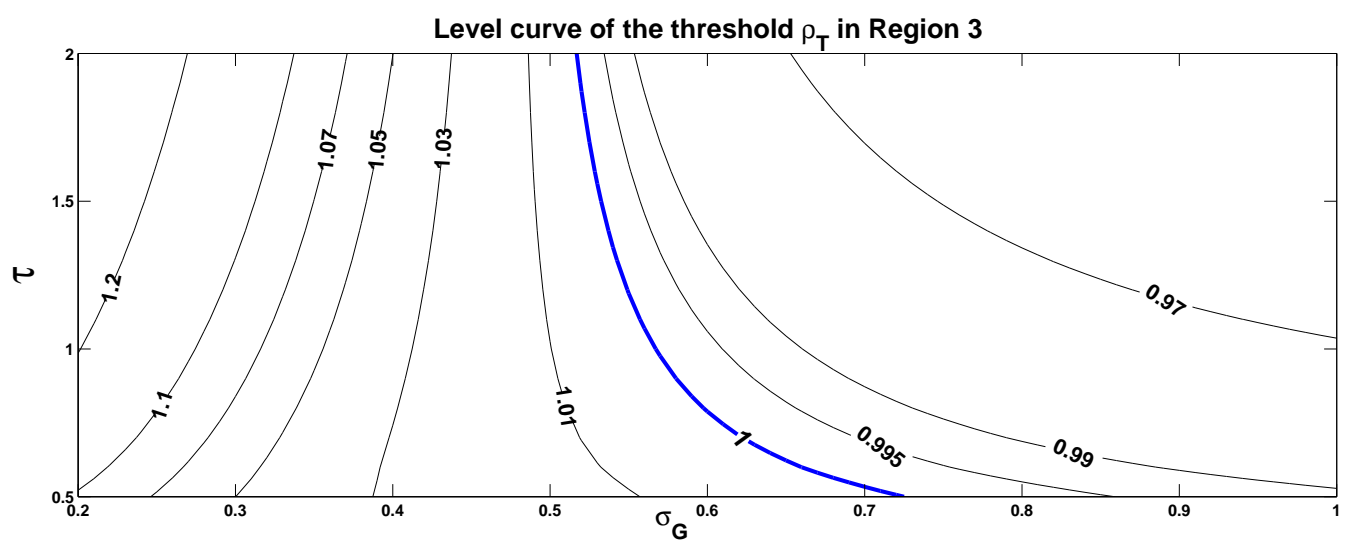

Figure 5: Level curve of the threshold $\rho_{T}$ illustrating that system (11)-(2) is liable to move from a savanna/forest state to a grassland state or to a multistability involving the grassland solution in relation to $\tau$ and $\sigma_{G}$ variations. Recall that the grassland solution is stable (resp. unstable) whenever $\rho_{T}$ is lower (resp. greater) than unity.

Since for parameters values in Table 7 and according to our estimation of $\sigma_{N S}$ one has

- $\mathcal{R}_{T}^{G}<1, \rho_{T}^{G}<1$,

- $\mathcal{R}_{G}^{0}>1$ then,

$$
\rho_{G}^{0}>1 \Longleftrightarrow \tau>0.2291
$$

Values of $\tau$ are not expected to be under 0.5 (i.e. 2 fires per year), a minimum which corresponds to sub-equatorial climates with two dry seasons. We therefore consider that this condition is always fulfilled.

Therefore, one can deduce that figure 5 illustrates either case 8, 9 or case 10 of Table 3.4.

The previous analysis highlighted the importance of the grass vs. sensitive tree competition parameter, $\sigma_{G}$, the non sensitive tree vs. grass competition parameter, $\sigma_{N S}$, and the fire return time, $\tau$, in controlling the outcomes of the IFAC model. Comparing to results of Region 2 (in terms of having the forest, the grassland or the savanna as reachable solution), one note that the IFAC model fairly has the same outcomes in Region 3 as in Region 2. Figure 5 further show that for low values of $\sigma_{G}$, say less than $0.6, \tau$ seems to have very limited influence. It is stronger for large values for which increases in $\tau$ make $\rho_{T}$ decreases under 1 and therefore destabilize the grassland solution.

In summary, in Region 3, we observed that the fire return time along with the grass vs. sensitive tree competition parameter $\sigma_{G}$ and the non sensitive tree vs. grass competition parameter $\sigma_{N S}$ strongly influence the outcome of the IFAC model. Indeed, depending on these parameters variations and values, the IFAC can converge either to a grassland state, to a savanna state, to a forest state or to a multistability involving forest state and either grassland or savanna while environmental conditions in this Region would systematically allow forests in the absence of fire (Staver and Levin (2012) [44]). Therefore to favor a forest state in Region 3, one could implement policies in order to have relatively large fire return time (says a fire frequency, f, lower than one fire per year: $f<1$ ) and vice versa if tracts of savanna are to be kept against forest encroachment as habitats of large grazing mammals. 


\section{Conclusion}

In this work, we presented and analyzed a new kind of mathematical model for tree-grass interactions in savanna ecosystems either fire prone or not. It is an extension of a continuoustime model, called the COFAC model, studied in Yatat et al. (2014) [52]. The model presented here, that we call the IFAC model, is based on a system featuring impulsive differential equations and thereby aims to acknowledge the discrete nature of fire events. The analytical study of the IFAC reveals a desert equilibrium, a forest equilibrium and two periodic solutions: the grassland periodic solution and the savanna periodic solution. The analytical study also reveals seven ecological thresholds $\left(\mathcal{R}_{T}^{0}, \mathcal{R}_{G}^{0}, \rho_{G}^{0}, \mathcal{R}_{G}^{T}, \mathcal{R}_{T}^{G}, \rho_{T}^{G}, \rho_{T}\right)$. These thresholds define in parameter space regions of monostability, bistability also found with the models of Accatino et al. (2010) [3], De Michele et al. (2011) [19], Yatat et al. (2014) [52]. They also define regions of tristability as in Yatat et al. (2014) [52] with respect to the equilibria (desert and forest) and periodic solutions (grassland and savanna). The specificity of IFAC is to also present periodic behaviors which depict fluctuations in woody and/or grassy biomass and cannot be yielded by fully continuous time models. Therefore, in case of transition from a vegetation type (forest, savanna or grassland) to another, the change is done progressively which is more ecologically meaningful than the abrupt changes observed for fires-continuous models such as in Accatino et al. (2010) [3], De Michele et al. (2011) [19] and Yatat et al. (2014) [52]. Such abrupt changes have been criticized as unrealistic by Accatino et al. (2013) [2] (see also Beckage et al. (2011) [12]) who advocated stochastic models in lieu of continuous time formulation of fire impact on vegetation. The present approach however demonstrates that a more realistic modeling of fire can be introduced within the framework of continuous time models while keeping the potential for analytical exploration of the main outcomes of the model. Something which is not possible with the aforementioned fully stochastic models.

As in Yatat et al. (2014) [52], we found that the competition parameters $\sigma_{G}$ which expresses the asymmetric competition exerted by grasses on sensitive trees (shading and soil resource preemption) and $\sigma_{N S}$ that expresses the asymmetric competition of non sensitive trees on grasses (shading and soil resource preemption) are bifurcation parameters of the IFAC model along with fire frequency (which strongly influence the convergence outcomes of the IFAC model). The analytical study of the IFAC model also reveals three particular values $\tau^{\star}, \sigma_{N S}^{\star}$ and $\sigma_{G}^{\star}$ (see relation (83), Page 42) that delimit regions of stability/instability or forest and grassland solutions in relation to $\tau, \sigma_{G}$ and $\sigma_{N S}$ respectively (see relations (13), Page 8 and (17), Page 9). Moreover, considering three ecological biomass production zones indexed by fires frequency and by carrying capacities of both trees and grasses biomass, allows us to point out several scenarios for IFAC convergence that depend on $\sigma_{G}, \sigma_{N S}$ and $\tau$ values and variations. These outcomes of the IFAC model are qualitatively in agreement with results of Baudena et al. (2014) [11, Staver et al. (2011) [43], February et al. (2013) [25], Accatino et al. (2010) [3], Couteron \& Kokou (1997) [17], Staver \& Levin (2012) [44]. Distinguishing three ecological zones allowed us to verify in which contexts the possible bifurcation parameters are actually influential or not. This analysis highlighted the pervasiveness of $\sigma_{N S}$ (i.e. the depressive or facilitation effect of grown-up trees on grasses) in all the three zones. It also emphasized the influence of $\sigma_{G}$ (i.e. depressive effect of grasses on small trees) in the two zones (2 and 3 ) with sufficient rainfall to allow medium to high grass production. In these two zones, and especially in zone 3, the fire return time $(\tau)$ appeared also influential. 
As already mentioned by Yatat et al. (2014) [52], and verified here for the IFAC model, the competition parameters $\sigma_{G}$ and $\sigma_{N S}$ which embody direct tree-grass interactions deserve an increased interest and should be the focus of adhoc observations and experiments as to better assess their ranges of variation in the different ecological regions.

Although the IFAC model presented in this work and the COFAC model presented in Yatat et al. (2014) [52] qualitatively display strong similarities, the IFAC model is richer. Indeed modelling fire events as pulse phenomena leads to a relaxation of stability conditions of both forest and grassland solutions and it increases parameters ranges for which bistability situations involving forest, grassland and savanna can occur. This particular property of the IFAC model may explain, along with bifurcation parameters and its periodic outcomes, many changes in tree-grass interactions in fire-prone ecosystems. Thanks to this particular property, the IFAC model, which moreover displays periodic outcomes and bifurcations according to well-identified parameters, is able to account for many dynamical scenarios observed in savanna-like ecosystems from the fringes of the desert to the boundary of the wet forest.

\section{Acknowledgements}

The first author is grateful to the French governement and the French Embassy in Yaoundé (Cameroon) for their support (SCAC fund) during the preparation of this manuscript.

\section{References}

[1] L. Abbadie, J. Gignoux, X. Le Roux and M. Lepage, Lamto: structure, functioning, and dynamics of a Savanna Ecosystem. Eco. Stu. Spinger, 2006.

[2] F. Accatino and C. De Michele, Humid savanna-forest dynamics: a matrix model with vegetation-fire interactions and seasonality. Eco. Mod. 265, pp. 170-179, 2013.

[3] F. Accatino, C. De Michele, R. Vezzoli, D. Donzelli and R. Scholes, Tree-grass coexistence in savanna: interactions of rain and fire.J. Theor. Biol. 267, pp. 235-242, 2010.

[4] R. Anguelov, Y. Dumont and J.M.-S. Lubuma, On nonstandard finite difference schemes in biosciences. AIP Conf. Proc. 1487, pp. 212-223, 2012.

[5] R. Anguelov, Y. Dumont, J.M.-S. Lubuma and E. Mureithi, Stability Analysis and Dynamics Preserving Non-Standar Finite Difference Schemes for Malaria Model, Mathematical Population Studies, 20 (2), pp. 101-122, 2013.

[6] R. Anguelov, Y. Dumont, J.M.-S. Lubuma and M. Shillor, Dynamically consistent nonstandard finite difference schemes for epidemiological Models. Journal of Computational and Applied Mathematics, 255, pp. 161-182, 2014.

[7] P. Augier, C. Lett and J.C. Poggiale, Modélisation mathématique en écologie. Cours et exercices corrigés. Dunod, Paris, 2010.

[8] D.D. Bainov and P.S. Simeonov, Impulsive Differential Equations: Asymptotic properties of the solutions. World scientific publishing Co. 1995. 
[9] N. Barbier, P. Couteron, R. Lefever, V. Deblauwe and O. Lejeune, Spatial decoupling of facilitation and competition at the origin of gapped vegetation patterns. Ecol. 89, pp. 1521-1531, 2008.

[10] M. Baudena, F. D'Andrea and A. Provenzale, An idealized model for tree-grass coexistence in savannas: the role of life stage structure and fire disturbances. J. Ecol. 98, pp. 74-80, 2010.

[11] M. Baudena, S.C. Dekker, P.M. van Bodegom, B. Cuesta, S.I. Higgins, V. Lehsten, C.H. Reick, M. Rietkerk, S. Scheiter, Z. Yin, M.A. Zavala and V. Brovkin, Forest, Savannas and grasslands: bridging the knowledge gap between ecology and dynamic global vegetation models. Biogeosciences Discuss., 11, pp. 9471-9510, 2014.

[12] B. Beckage, L.J. Gross and W.J. Platt, Grass feedbacks on fire stabilize savannas. Eco. Mod. 222, pp. 2227-2233, 2011.

[13] A. J. Belsky, R. G. Amundson, J. M. Duxbury, S. J. Rika, A. R. Ali and S. M. Mwonga, The effects of trees on their physical, chemical, and biological environment in a semi-arid savanna in Kenya. J. Appl. Ecol. 26: pp. 1005-1024, 1989.

[14] W.J. Bond, G.F. Midgley and F.I. Woodward, What controls South African vegetationclimate or fire? S. Afr. J. Bot. 69, pp. 79-91, 2003.

[15] H. Breman and J.J. Kessler, Woody plants in agroecosystems of semi-arid regions. With an emphasis on the Sahelian countries. Advanced series in Agricultural 23, SpringerVerlag, Berlin. 1995.

[16] Y. Chen, Z. Liu and M. Haque, Analysis of a Leslie-Gower-type prey-predator model with periodic impulsive perturbations. Com. Non. Sci. Numer. Simulat. 14, pp. 34123423, 2009.

[17] P. Couteron and K. Kokou, Woody vegetation spatial patterns in a semi-arid savanna of Burkina Faso, West Africa. Plant Ecol. 132, pp. 211-227, 1997.

[18] C. Dai, M. Zhao and L. Chen, Dynamic Complexity of an Ivlev-Type Prey-Predator System with Impulsive State Feedback Control. J. App. Math. Article ID 534276, pp. $17,2012$.

[19] C. De Michele, F. Accatino, R. Vezzoli and R.J. Scholes, Savanna domain in the herbivores-fire parameter space exploiting a tree-grass-soil water dynamic model. J. Theor. Biol. 289, pp. 74-82, 2011.

[20] P. D'Odorico, F. Laio and L.A. Ridolfi, probabilistic analysis of fire-induced tree-grass coexistence in savannas. The American Naturalist, 167, pp. E79-E87, 2006.

[21] D. D'Onofrio, Stability properties of pulse vaccination strategy in SEIR epidemic model. Mathematical Biosciences, 179, pp. 57-72, 2002.

[22] Y. Dumont, J.C. Russell, V. Lecomte and M. Le Corre, Conservation of endangered endemic seabirds within a multi-predator context: The Barau's petrel in Réunion island. Natural Ressource Modelling, 23, pp. 381-436, 2010. 
[23] Y. Dumont and J.M. Tchuenche, Mathematical Studies on the Sterile Insect Technique for the Chikungunya Disease and Aedes albopictus. Journal of Mathematical Biology, 65 (5), pp. 809-854, 2012.

[24] C. Favier, J. Aleman, L. Bremond, M.A. Dubois, V. Freycon and J.M. Yangakola, Abrupt shifts in African savanna tree cover along a climatic gradient, Global Ecology and Biogeography, 21, pp. 787-797, 2012.

[25] E.C. February, S.I. Higgins, W.J. Bond and L. Swemmer, Influence of competition and rainfall manipulation on the growth responses of savanna trees and grasses. Ecology. 94 (5), pp. 1155-1164, 2013.

[26] C. Fernandez-Oto, M. Tlidi, D. Escaff and M. G. Clerc, Strong interaction between plants induces circular barren patches: fairy circles. Phil. Trans. R. Soc. A 28 October 2014 vol. 372 no. 202720140009.

[27] R. E. Gaines and J. Mawhin. Coincidence degree and nonlinear differential equations. Lecture Notes in Mathematic, Vol. 568, Springer, 1977.

[28] J. Gignoux, Modélisation de la coexistence herbes-arbres en savane, PhD Thesis, Paris INA-PG, 1994.

[29] S.I. Higgins, W.J. Bond, W.S.W. Trollope and R.J. Williams, Physically motivated empirical models for the spread and intensity of grass fires, Int. J. Wildland Fire, 17, pp. 595-601, 2008.

[30] A. Kang, Y. Xue and Z. Jin, Dynamic behavior of an eco-epidemic system with impulsive birth, J. Math. Anal. Appl., 345, pp. 783-795, 2008.

[31] V.F. Langevelde, C. van de Vijver, L. Kumar, J. van de Koppel, N. de Ridder, J. van Andel, et al., Effects of fire and herbivory on the stability of savanna ecosystems. Ecology, 84 (2), pp. 337-350, 2003.

[32] R. Lefever, N. Barbier, P. Couteron and O. Lejeune, Deeply gapped vegetation patterns: On crown/root allometry, criticality and desertification. Jour. Theo. Ecol., 261, pp. 194209, 2009.

[33] M. Liu, Z. Jin, M. Haque, An impulsive predator-prey model with communicable disease in the prey species only, Nonlin. Ana. Real World App. 10, pp. 3098-3111, 2009.

[34] O. Maurin, T.J. Davies, J.E. Burrows, B.H. Daru, K. Yessoufou, A.M. Muasya, M. Van der Bank and J.W. Bond. Savanna fire and the origins of the underground forests of Africa. New Phytologist. pp. 1-14, 2014.

[35] J.C. Menaut and J. César, Structure and primary productivity of Lamto savannas, Ivory Coast. Ecology. 60, pp. 1197-1210, 1979.

[36] P. Mordelet and J.C. Menaut, Influence of trees on above-ground production dynamics of grasses in a humid savanna. Journal of Vegetation Science. 6, pp. 223-228, 1995. 
[37] A. Moustakas, W. E. Kunin, T. C. Cameron and M. Sankaran. Facilitation or Competition? Tree Effects on Grass Biomass across a Precipitation Gradient. PLoS ONE 8(2): e57025, 2013.

[38] F.W.T. Penning de Vries and M.A. Djiteye. La productivité des paturages sahéliens. Une étude des sols, des végétations et de l'exploitation de cette ressource naturelle. PUDOC, Wageningen, 1982.

[39] M. Sankaran, J. Ratnam and N. Hanan. Woody cover in African savannas: the role of resources, fire and herbivory. Global Ecology and Biogeography, 17, pp. 236-245, 2008.

[40] M. Sankaran, N.P. Hanan, R.J. Scholes, J. Ratnam, D.J. Augustine, B.S. Cade, J. Gignoux, S.I. Higgins, X. LeRoux, F. Ludwig, J. Ardo, F. Banyikwa, A. Bronn, G. Bucini, K.K. Caylor, M.B. Coughenour, A. Diouf, W. Ekaya, C.J. Feral, E.C. February, P.G.H. Frost, P. Hiernaux, H. Hrabar, K.L. Metzger, H.H.T. Prins, S. Ringrose, W. Sea, J. Tews, J. Worden and N. Zambatis. Determinants of woody covering African savannas. Nature, 438, pp. 846-849, 2005.

[41] I.P.J. Smit, G. Asner, N. Govender, T. Kennedy-Bowdoin, D. Knapp and J. Jacobson, Effects of fire on woody vegetation structure in African savanna. Ecological Applications, 20 (7), pp. 1865-1875, 2010.

[42] Y. Sonntag, Topologie et analyse fonctionnelle. Cours de Licence avec 240 exercices et 30 problèmes corrigés. Ellipses, 1997.

[43] A.C. Staver, S. Archibald and S. Levin, Tree cover in sub-Saharan Africa: Rainfall and fire constrain forest and savanna as alternative stable states. Ecology. 92(5), pp. 1063-1072, 2011.

[44] A.C. Staver and S. Levin, Integrating theoretical climate and fire effects on savanna and forest systems. Am. Nat. 180(2), 2012.

[45] A. Tchuinte, J. J. Tewa, P. Couteron, S. Bowong and Y. Dumont, A Generic Modeling of Fire Impact in a Tree-Grass Savanna Model, Biomath 3 (2014), 1407191.

[46] D. Tilman, Competition and biodiversity in spatially structured habitats. Ecology, 75, pp. 2-16, 1994.

[47] W. R. Tschinkel, The Life Cycle and Life Span of Namibian Fairy Circles, PLoS ONE 7(6): e38056, 2012.

[48] C.A. Van de Vijver, Foley and H. Olff, Changes in the woody component of an East African savanna during 25 years. Journal of Tropical Ecology 15, pp. 545-564, 1999.

[49] J.L. Wakeling, A.C. Staver and W.J. Bond, Simply the best: the transition of savanna saplings to trees. Oikos, 120, pp. 1448-1451, 2011.

[50] B. Walker, D. Ludwig, C.S. Holling and R.M. Peterman, Stability of semi-arid savanna grazing systems. Journal of Ecology, 69, pp. 473-498, 1981. 
[51] J. F. Weltzin, and M. B. Coughenour, Savanna tree influence on understory vegetation and soil nutrients in northwestern Kenya. J. Veg. Sci. 1: 325-334, 1990.

[52] V. Yatat, Y. Dumont, J. J. Tewa, P. Couteron and S. Bowong, Mathematical Analysis of a Size Structured Tree-Grass Competition Model for Savanna Ecosystems, Biomath 3 (2014), 1404212.

\section{Appendix A: Proof of Lemma 3}

Defining

$$
\begin{array}{ll}
T_{S}(t) & =x(t), \\
T_{N S}(t) & =y(t), \\
G(t) & =G^{*}(t)+z(t),
\end{array}
$$

where $x(t), y(t)$ and $z(t)$ are small perturbations. Every solution of the linearized equations can be written as

$$
\left(\begin{array}{l}
x(t) \\
y(t) \\
z(t)
\end{array}\right)=\Phi(t)\left(\begin{array}{c}
x(0) \\
y(0) \\
z(0)
\end{array}\right)
$$

Here $\Phi$ is a fundamental matrix and satisfies,

$$
\begin{aligned}
\frac{d \Phi(t)}{d t} & =D F\left(0 ; 0 ; G^{*}(t)\right) \Phi(t) \\
& =\left(\begin{array}{ccc}
\gamma_{S}-\left(\mu_{S}+\omega_{S}+\sigma_{G} G^{*}(t)\right) & \gamma_{N S} & 0 \\
\omega_{S} & -\mu_{N S} & 0 \\
0 & -\sigma_{N S} G^{*}(t) & \gamma_{G}-2 \frac{\gamma_{G}}{K_{G}} G^{*}(t)-\mu_{G}
\end{array}\right) \Phi(t)
\end{aligned}
$$

and $\Phi(0)=I d_{\mathbb{R}^{3}}$. Moreover the resetting impulsive condition of system (11)-(2) becomes,

$$
\left(\begin{array}{c}
x\left(n \tau^{+}\right) \\
y\left(n \tau^{+}\right) \\
z\left(n \tau^{+}\right)
\end{array}\right)=\left(\begin{array}{ccc}
1-\eta_{S} w\left(G^{*}(\tau)\right) & 0 & 0 \\
0 & 1 & 0 \\
0 & 0 & 1-\eta_{G}
\end{array}\right)\left(\begin{array}{c}
x(n \tau) \\
y(n \tau) \\
z(n \tau)
\end{array}\right)
$$

A monodromy matrix $\mathbf{M}$ of system (11) - (2), is:

$$
\mathbf{M}=\left(\begin{array}{ccc}
1-\eta_{S} w\left(G^{*}(\tau)\right) & 0 & 0 \\
0 & 1 & 0 \\
0 & 0 & 1-\eta_{G}
\end{array}\right) \Phi(\tau)
$$

with

$$
\Phi(t)=\exp \left(\int_{0}^{t} D F\left(0 ; 0 ; G^{*}(s)\right) d s\right) .
$$

Moreover using Lemma 2, a direct computations leads

$$
\int_{0}^{\tau} D F\left(0 ; 0 ; G^{*}(s)\right) d s=\left(\begin{array}{ccc}
D F^{(1)} & D F^{(2)} & 0 \\
D F^{(3)} & D F^{(4)} & 0 \\
0 & D F^{(5)} & D F^{(6)}
\end{array}\right)
$$


where

$$
\begin{aligned}
D F^{(1)} & =\left(\gamma_{S}-\left(\mu_{S}+\omega_{S}\right)\right) \tau-\sigma_{G} \int_{0}^{\tau} G^{*}(t) d t, \\
D F^{(2)} & =\gamma_{N S} \tau \\
D F^{(3)} & =\omega_{S} \tau \\
D F^{(4)} & =-\mu_{N S} \tau \\
D F^{(5)} & =-\sigma_{N S} \int_{0}^{\tau} G^{*}(t) d t, \\
D F^{(6)} & =-\mu_{G}\left(\mathcal{R}_{G}^{0}-1\right) \tau-2 \ln \left(1-\eta_{G}\right) .
\end{aligned}
$$

Consider the sub-matrix $\mathbf{B}$ defined as follow:

$$
\mathbf{B}=\left(\begin{array}{ll}
D F^{(1)} & D F^{(2)} \\
D F^{(3)} & D F^{(4)}
\end{array}\right)
$$

Recall that eigenvalues of the matrix $\mathbf{B}$ are root of the quadratic equation

$$
\lambda^{2}-\operatorname{trace}(\mathbf{B}) \lambda+\operatorname{det}(\mathbf{B})=0
$$

and to characterize real part of eigenvalues of matrix $\mathbf{B}$, following Routh-Hurwitz criterium (see Section 1.3.5 Page 72 of Augier et al. (2010) [7]), one need only to study the sign of $\operatorname{trace}(\mathbf{B})$ and $\operatorname{det}(\mathbf{B})$. Let

$$
\mathcal{A}=\operatorname{tr}(\mathbf{B})=\gamma_{S} \tau\left(1-\frac{1}{\mathcal{R}}\right)
$$

where

$$
\mathcal{R}=\frac{\gamma_{S}}{\mu_{S}+\omega_{S}+\mu_{N S}+\sigma_{G} G_{i n t}}>0 .
$$

Thus, if $\mathcal{R}<1$ then $\mathcal{A}<0$.

Moreover, let

$$
\mathcal{B}=\operatorname{det}(\mathbf{B})=\tau \mu_{N S}\left(\left(\mu_{S}+\omega_{S}\right) \tau+\sigma_{G} G_{i n t}\right)\left(1-\mathcal{R}_{G}^{T}\right)
$$

where

$$
\mathcal{R}_{G}^{T}=\frac{\gamma_{S} \mu_{N S}+\omega_{S} \gamma_{N S}}{\mu_{N S}\left(\mu_{S}+\omega_{S}\right)+\sigma_{G} \mu_{N S} G_{i n t}}>0 .
$$

Thus, if $\mathcal{R}_{G}^{T}<1$ then $\mathcal{B}>0$.

Moreover, one also has $\mathcal{R}<\mathcal{R}_{G}^{T}$.

Therefore, if $\mathcal{R}_{G}^{T}<1$ then $s(\mathbf{B})<0$, where $s$ denotes the stability modulus (i.e. the maximum of the real part of eigenvalues).

From expressions (40) and (41) we deduced that eigenvalues $\xi_{1}, \xi_{2}$ and $\xi_{3}$ of the monodromy matrix $\mathbf{M}$ are

$$
\begin{aligned}
& \xi_{1}=\left(1-\eta_{S} w\left(G^{*}(\tau)\right)\right) e^{\lambda_{1}} \\
& \xi_{2}=e^{\lambda_{2}} \\
& \xi_{3}=\frac{e^{-\mu_{G}\left(\mathcal{R}_{G}^{0}-1\right) \tau}}{1-\eta_{G}}
\end{aligned}
$$

where $\lambda_{1}, \lambda_{2} \in \operatorname{sp}(\mathbf{B})$.

Since $0<1-\eta_{S} w\left(G^{*}(\tau)\right) \leq 1$, if $\mathcal{R}_{G}^{T}<1$, then $0 \leq \xi_{1}<1$ and $0<\xi_{2}<1$.

Moreover, since $\rho_{G}^{0}>1$ then $\xi_{3}<1$. Indeed,

$$
\xi_{3}<1 \Leftrightarrow e^{-\mu_{G}\left(\mathcal{R}_{G}^{0}-1\right) \tau}<1-\eta_{G} \Leftrightarrow 1<\left(1-\eta_{G}\right) e^{\mu_{G}\left(\mathcal{R}_{G}^{0}-1\right) \tau} \Leftrightarrow \rho_{G}^{0}>1 .
$$


Finally we deduce that the grassland periodic solution $E_{G}=\left(0 ; 0 ; G^{*}(t)\right)$ is locally asymptotically stable if $\mathcal{R}_{G}^{T}<1$ or $\left(\mathcal{R}_{G}^{T}>1\right.$ and $\left.\rho_{T}<1\right)$, is locally stable if $\left(\mathcal{R}_{G}^{T}>1\right.$ and $\left.\rho_{T}=1\right)$ and is unstable if $\left(\mathcal{R}_{G}^{T}>1\right.$ and $\left.\rho_{T}>1\right)$. This ends the proof.

\section{Appendix B: Proof of Theorem 1}

$\star$ Case 1: $\mu_{G}>0$.

Solution $G$ of system (11)-(2) satisfy

$$
\begin{aligned}
G^{\prime}(t) & \leq \gamma_{G}\left(1-\frac{1}{\mathcal{R}_{G}^{0}}\right) G(t) \\
G\left(t_{k}^{+}\right) & =\left(1-\eta_{G}\right) G\left(t_{k}\right) .
\end{aligned}
$$

From Lemma 1.3 page 15 in [8] we deduce that

$$
G(t) \leq G(0)\left(\prod_{0 \leq t_{k}<t}\left(1-\eta_{G}\right)\right) \exp \left(\gamma_{G}\left(1-\frac{1}{\mathcal{R}_{G}^{0}}\right) t\right) .
$$

Thus, for $\mathcal{R}_{G}^{0}<1$ we have $\lim _{t \rightarrow+\infty} G(t)=0$ and solutions $T_{S}$ and $T_{N S}$ of system (11)-(2) satisfy

$$
\left\{\begin{aligned}
T_{S}^{\prime} & =\left(\gamma_{S} T_{S}+\gamma_{N S} T_{N S}\right)\left(1-\frac{T_{S}+T_{N S}}{K_{T}}\right)-T_{S}\left(\mu_{S}+\omega_{S}\right), \\
T_{N S}^{\prime} & =\omega_{S} T_{S}-\mu_{N S} T_{N S} .
\end{aligned}\right.
$$

System (48) does not admit periodic solution (see Appendix B in Yatat et al. (2014) [52]), thus using the jacobian matrix of system (48) we deduce that

- if $\mathcal{R}_{T}^{0}<1$ then, $\left(T_{S}, T_{N S}\right) \rightarrow(0,0)$,

- if $\mathcal{R}_{T}^{0}>1$ then, $\left(T_{S}, T_{N S}\right) \rightarrow\left(\bar{T}_{S}, \bar{T}_{N S}\right)$, where $\left(\bar{T}_{S}, \bar{T}_{N S}\right)$ are given in (15).

At the end, we deduce that if $\mathcal{R}_{T}^{0}<1$ and $\mathcal{R}_{G}^{0}<1$ then, the desert equilibrium $E_{0}$ is GAS i.e., point 1 of Theorem 1 holds. The forest equilibrium $E_{T}$ is GAS whenever $\mathcal{R}_{T}^{0}>1$ and $\mathcal{R}_{G}^{0}<1$ i.e., point 2 of Theorem 1 holds.

Now suppose that $\mathcal{R}_{T}^{0}<1$ and $\mathcal{R}_{G}^{0}>1$. Solutions $T_{S}$ and $T_{N S}$ of system (1)-(2) satisfy

$$
\begin{aligned}
&\left\{\begin{array}{rl}
\frac{d T_{S}}{d t} \leq & \left(\gamma_{S} T_{S}+\gamma_{N S} T_{N S}\right)\left(1-\frac{T_{S}+T_{N S}}{K_{T}}\right)-T_{S}\left(\mu_{S}+\omega_{S}\right), \\
\frac{d T_{N S}}{d t} \leq & \omega_{S} T_{S}-\mu_{N S} T_{N S},
\end{array} \quad t \neq t_{k}\right. \\
&\left\{\begin{array}{l}
T_{S}\left(t_{k}^{+}\right) \leq T_{S}\left(t_{k}\right), \\
T_{N S}\left(t_{k}^{+}\right) \leq T_{N S}\left(t_{k}\right), \quad t=t_{k} \quad t_{k+1}=t_{k}+\tau .
\end{array}\right.
\end{aligned}
$$

Let consider the upper system

$$
\left\{\begin{array}{l}
\frac{d u}{d t}=\left(\gamma_{S} u+\gamma_{N S} v\right)\left(1-\frac{u+v}{K_{T}}\right)-u\left(\mu_{S}+\omega_{S}\right) \\
\frac{d v}{d t}=\omega_{S} u-\mu_{N S} v
\end{array}\right.
$$


Since $\mathcal{R}_{T}^{0}<1,(u(t), v(t)) \rightarrow(0,0)$. Thus $\left(T_{S}(t), T_{N S}(t)\right) \rightarrow(0,0)$. Furthermore, solution $G$ of system (1)-(2) admits as limiting system

$$
\left\{\begin{array}{lll}
\frac{d G}{d t} & =\gamma_{G}\left(1-\frac{G}{K_{G}}\right) G-\mu_{G} G & t \neq t_{k} \\
G\left(t_{k}^{+}\right)=\left(1-\eta_{G}\right) G\left(t_{k}\right) & t=t_{k} .
\end{array}\right.
$$

System (52) admits at most two solutions: the trivial solution, 0, which always exists and the periodic solution $G^{*}(t)$ which is ecologically meaningful if $\rho_{G}^{0}>1$ where $G^{*}(t)$ is given by (81). Now we turn to check stability results of solutions of system (52) through small perturbations approach and Floquet's theory.

- Setting $G(t)=x(t)$ where $x$ is a small perturbation and verify $x(t)=\phi(t) x_{0}$, where $\phi$ verify

$$
\phi^{\prime}(t)=\mu_{G}\left(\mathcal{R}_{G}^{0}-1\right) \phi(t)
$$

and $\phi(0)=1$. The resulting impulsive condition becomes

$$
x\left(n T^{+}\right)=\left(1-\eta_{G}\right) x(n T) .
$$

Following the Floquet's theory, the zero equilibrium is locally asymptotically stable if

$$
\lambda_{0}=\left(1-\eta_{G}\right) e^{\mu_{G}\left(\mathcal{R}_{G}^{0}-1\right) \tau}<1 .
$$

Since

$\mathcal{R}_{G}^{0}>1, \lambda_{0}<1$ if and only if $\left(1-\eta_{G}\right) e^{\mu_{G}\left(\mathcal{R}_{G}^{0}-1\right) \tau}<1$, i.e. $\rho_{G}^{0}<1$.

Moreover, for $\rho_{G}^{0}<1$ the positive solution $G^{*}(t)$ is undefined then the desert equilibrium is globally asymptotically stable. Finally, we deduce that the desert solution $(0,0,0)$ is globally asymptotically stable whenever $\mathcal{R}_{T}^{0}<1, \mathcal{R}_{G}^{0}>1$ and $\rho_{G}^{0}<1$. Point 3 of Theorem 1 holds.

- Now, setting $G(t)=G^{*}(t)+x(t)$ where $x$ is a small perturbation and verify $x(t)=$ $\phi(t) x_{0}$, where $\phi$ verify

$$
\phi^{\prime}(t)=\left[\mu_{G}\left(\mathcal{R}_{G}^{0}-1\right)-\frac{2 \gamma_{G}}{K_{G}} G^{*}(t)\right] \phi(t)
$$

and $\phi(0)=1$. The resulting impulsive condition becomes

$$
x\left(n T^{+}\right)=\left(1-\eta_{G}\right) x(n T) .
$$

According to the Floquet's theory, solution $G^{*}(t)$ is locally asymptotically stable if

$$
\lambda_{G^{*}}=\left(1-\eta_{G}\right) \exp \left\{\mu_{G}\left(\mathcal{R}_{G}^{0}-1\right) \tau-\frac{2 \gamma_{G}}{K_{G}} \int_{n \tau}^{(n+1) \tau} G^{*}(t) d t\right\}<1 .
$$

Following Lemma 2,

$$
\int_{n \tau}^{(n+1) \tau} G^{*}(s) d s=\frac{K_{G}}{\gamma_{G}}\left\{\ln \left(1-\eta_{G}\right)+\mu_{G}\left(\mathcal{R}_{G}^{0}-1\right) \tau\right\} .
$$


Thus

$$
\begin{aligned}
\lambda_{G^{*}} & =\left(1-\eta_{G}\right) \exp \left\{-\mu_{G}\left(\mathcal{R}_{G}^{0}-1\right) \tau-2 \ln \left(1-\eta_{G}\right)\right\} \\
& =\exp \left\{-\mu_{G}\left(\mathcal{R}_{G}^{0}-1\right) \tau-\ln \left(1-\eta_{G}\right)\right\} .
\end{aligned}
$$

Since $\rho_{G}^{0}>1$, we have:

$$
\begin{aligned}
\rho_{G}^{0}>1 & \Leftrightarrow\left(1-\eta_{G}\right) \exp \left\{\mu_{G}\left(\mathcal{R}_{G}^{0}-1\right) \tau\right\}>1 \\
& \Leftrightarrow \ln \left(1-\eta_{G}\right)>-\mu_{G}\left(\mathcal{R}_{G}^{0}-1\right) \tau \\
& \Leftrightarrow-\mu_{G}\left(\mathcal{R}_{G}^{0}-1\right) \tau-\ln \left(1-\eta_{G}\right)<0
\end{aligned}
$$

and we deduce

$$
\lambda_{G^{*}}<1 .
$$

Thus solution $G^{*}(t)$ of (52) is globally asymptotically stable because the zero solution, in this case, is unstable. Finally, we deduce that the grassland periodic solution $\left(0,0, G^{*}(t)\right)$ is globally asymptotically stable whenever $\mathcal{R}_{T}^{0}<1, \mathcal{R}_{G}^{0}>1$ and $\rho_{G}^{0}>1$. Point 4 of Theorem 1 holds.

$\star$ Case 2: $\mu_{G}=0$.

The proof of points (i) and (ii) of Theorem 1 is fairly the same as the proof of points 3 and 4. Indeed we first set, only in System (52),$\mu_{G}=0$ and next, we substitute $\mu_{G}\left(\mathcal{R}_{G}^{0}-1\right)$ by $\gamma_{G}$ in the rest of the proof.

Solution $G$ of system (11)-(2) satisfy

$$
\left\{\begin{array}{lll}
\frac{d G}{d t} & \leq \gamma_{G}\left(1-\frac{G}{K_{G}}\right) G & t \neq t_{k} \\
G\left(t_{k}^{+}\right)=\left(1-\eta_{G}\right) G\left(t_{k}\right) & t=t_{k} .
\end{array}\right.
$$

Since $\rho_{G}^{0}=\left(1-\eta_{G}\right) \exp \left\{\gamma_{G} \tau\right\}<1$, it follows that $G(t) \longrightarrow 0$. Therefore, solutions $T_{S}$ and $T_{N S}$ of (11)-(2) satisfy system (48). Since $\mathcal{R}_{T}^{0}<1$, one has $\left(T_{S}, T_{N S}\right) \longrightarrow\left(\bar{T}_{S}, \bar{T}_{N S}\right)$. Point (iii) of Theorem 1 holds.

\section{Appendix C: Proof of Theorem 2}

Taking new variables $T_{S}(t)=e^{x(t)}, T_{N S}(t)=e^{y(t)}, G(t)=e^{z(t)}$ then system (11) - (2) becomes,

$$
\left\{\begin{aligned}
\dot{x}(t) & =-\omega_{S}-\mu_{S}-\sigma_{G} e^{z}+\left(\gamma_{S}+\gamma_{N S} e^{y} e^{-x}\right)\left(1-\frac{e^{x}+e^{y}}{K_{T}}\right), \quad t \neq t_{n} . \\
\dot{y}(t) & =-\mu_{N S}+\omega_{S} e^{x} e^{-y}, \quad t_{n+1}=t_{n}+\tau \\
\dot{z}(t) & =\gamma_{G}\left(1-\frac{e^{z}}{K_{G}}\right)-\sigma_{N S} e^{y}-\mu_{G} \\
x\left(t^{+}\right) & =x(t)+\ln \left(1-\eta_{S} w\left(e^{z}\right)\right), \quad t=t_{n} . \\
y\left(t^{+}\right) & =y(t), \quad n=0,1,2, \ldots \\
z\left(t^{+}\right) & =z(t)+\ln \left(1-\eta_{G}\right) .
\end{aligned}\right.
$$

Let $X=C^{1}\left([0, \tau], \mathbb{R}^{3}\right), Z=C^{1}\left([0, \tau], \mathbb{R}^{3}\right) \times C^{1}\left([0, \tau], \mathbb{R}^{3}\right)$ and for $u=(x, y, z) \in X$,

$$
\|u\|=\max _{t \in[0, \tau]}|x(t)|+\max _{t \in[0, \tau]}|y(t)|+\max _{t \in[0, \tau]}|z(t)| .
$$


Then $X, Z$ are Banach spaces when they are endowed with the above norm $\|\cdot\|$.

Let,

$$
L: \operatorname{Dom}(L) \subset X \rightarrow Z,\left(\begin{array}{c}
x \\
y \\
z
\end{array}\right) \rightarrow\left(\left(\begin{array}{c}
\dot{x} \\
\dot{y} \\
\dot{z}
\end{array}\right),\left(\begin{array}{c}
\Delta x\left(t_{n}\right) \\
\Delta y\left(t_{n}\right) \\
\Delta z\left(t_{n}\right)
\end{array}\right)\right)
$$

and

where

$$
N\left(\begin{array}{l}
x \\
y \\
z
\end{array}\right)=\left(N_{1}\left(\begin{array}{l}
x \\
y \\
z
\end{array}\right), N_{2}\left(\begin{array}{l}
x \\
y \\
z
\end{array}\right)\right)
$$

$$
\begin{gathered}
N_{1}\left(\begin{array}{l}
x \\
y \\
z
\end{array}\right)=\left(\begin{array}{c}
-\omega_{S}-\mu_{S}-\sigma_{G} e^{z}+\left(\gamma_{S}+\gamma_{N S} e^{y} e^{-x}\right)\left(1-\frac{e^{x}+e^{y}}{K_{T}}\right) \\
-\mu_{N S}+\omega_{S} e^{x} e^{-y} \\
\gamma_{G}\left(1-\frac{e^{z}}{K_{G}}\right)-\sigma_{N S} e^{y}-\mu_{G}
\end{array}\right), \\
N_{2}\left(\begin{array}{c}
x \\
y \\
z
\end{array}\right)=\left(\begin{array}{c}
\ln \left(1-\eta_{S} w\left(e^{z(\tau)}\right)\right) \\
0 \\
\ln \left(1-\eta_{G}\right)
\end{array}\right) .
\end{gathered}
$$

A direct computation leads to

$$
\operatorname{Ker} L=\left\{\left(\begin{array}{l}
x \\
y \\
z
\end{array}\right):\left(\begin{array}{l}
x \\
y \\
z
\end{array}\right)=\left(\begin{array}{l}
c_{1} \\
c_{2} \\
c_{3}
\end{array}\right) \in \mathbb{R}^{3}, t \in[0, \tau]\right\}
$$

and

$$
\operatorname{Im} L=\left\{\left(\left(\begin{array}{c}
l \\
m \\
n
\end{array}\right),\left(\begin{array}{c}
a \\
b \\
c
\end{array}\right)\right) \in Z:\left(\begin{array}{c}
\int_{0}^{\tau} l(t) d t+a=0 \\
\int_{0}^{\tau} m(t) d t+b=0 \\
\int_{0}^{\tau} n(t) d t+c=0
\end{array}\right)\right\} .
$$

Since $I m L$ is closed in $Z, L$ is a Fredholm mapping of index zero. Indeed,

$\operatorname{Index}(L)=\operatorname{dim}(\operatorname{Ker} L)-\operatorname{dim}(\operatorname{CoKer} L)=\operatorname{dim}(\operatorname{Ker} L)-(\operatorname{dim}(Z)-\operatorname{dim}(\operatorname{Im} L))=3-(6-3)=0$.

Thus following (Gaines and Mawhin (1977) [27], Page 12), there exist two continuous projectors $P$ and $Q$ such that the sequel $X \stackrel{P}{\longrightarrow}$ Dom $L \stackrel{L}{\longrightarrow} Z \stackrel{Q}{\longrightarrow} Z$ is exact i.e $\operatorname{ImP}=\operatorname{Ker} L$ and $\operatorname{Ker} Q=\operatorname{Im} L=\operatorname{Im}(I-Q)$. It suffices to choose

$P\left(\begin{array}{l}x \\ y \\ z\end{array}\right)=\left(\begin{array}{l}x(\tau) \\ y(\tau) \\ z(\tau)\end{array}\right)$ and $Q\left(\left(\begin{array}{c}l \\ m \\ n\end{array}\right),\left(\begin{array}{l}a \\ b \\ c\end{array}\right)\right)=\left(\frac{1}{\tau}\left(\begin{array}{c}\int_{0}^{\tau} l(s) d t+a \\ \int_{0}^{\tau} m(s) d t+b \\ \int_{0}^{\tau} n(s) d t+c\end{array}\right),\left(\begin{array}{l}0 \\ 0 \\ 0\end{array}\right)\right)$.

One can verify that $L P\left(\begin{array}{l}x \\ y \\ z\end{array}\right)=0_{X}$ and $Q L\left(\begin{array}{l}x \\ y \\ z\end{array}\right)=0_{Z}$.

Furthermore, the generalized inverse $K_{P}: \operatorname{Im} L \rightarrow \operatorname{KerP} \cap \operatorname{Dom}(L)$ of the map $L: \operatorname{Ker} P \cap$ $\operatorname{Dom}(L) \rightarrow \operatorname{ImL}$ is given by

$$
K_{P}\left(\left(\begin{array}{c}
l \\
m \\
n
\end{array}\right),\left(\begin{array}{c}
a \\
b \\
c
\end{array}\right)\right)=\left(\begin{array}{c}
\int_{0}^{t} l(s) d s+a \\
\int_{0}^{t} m(s) d s+b \\
\int_{0}^{\tau} n(s) d s+c
\end{array}\right) .
$$


Indeed, let $u=\left(u_{1}, u_{2}, u_{3}\right)^{T} \in \operatorname{Ker} P \cap \operatorname{Dom}(L),(g, r)=\left(\left(g_{1}, g_{2}, g_{3}\right),\left(r_{1}, r_{2}, r_{3}\right)\right) \in \operatorname{ImL}$, we have

$$
\begin{aligned}
K_{P} L(u(t)) & =K_{P}(\dot{u}, \Delta u) \\
& =\int_{0}^{t} \dot{u}(s) d s+\Delta u \\
& =u(t)-u(0)+u(0)-u(\tau) \\
& =u(t)-P(u) \\
& =u(t), \text { because } u \in \operatorname{Ker} P
\end{aligned}
$$

and

$$
\begin{aligned}
L K_{P}(g(t), r) & =L\left(\int_{0}^{t} g(s) d s+r\right) \\
& =\left(g(t),-\int_{0}^{\tau} g(t) d t\right) \\
& =(g(t), r) \text { because }(g, r) \in I m L
\end{aligned}
$$

Thus,

$$
Q N\left(\begin{array}{l}
x \\
y \\
z
\end{array}\right)=\left(\left(\begin{array}{l}
A_{1} \\
A_{2} \\
A_{3}
\end{array}\right),\left(\begin{array}{l}
0 \\
0 \\
0
\end{array}\right)\right)
$$

Furthermore,

$$
\begin{aligned}
K_{P}(I-Q) N\left(\begin{array}{l}
x \\
y \\
z
\end{array}\right) & =K_{P} N\left(\begin{array}{l}
x \\
y \\
z
\end{array}\right)-K_{P} Q N\left(\begin{array}{l}
x \\
y \\
z
\end{array}\right) \\
& =\left(\begin{array}{c}
B_{1} \\
B_{2} \\
B_{3}
\end{array}\right)-\left(\begin{array}{l}
C_{1} \\
C_{2} \\
C_{3}
\end{array}\right)+\left(\begin{array}{c}
D_{1} \\
D_{2} \\
D_{3}
\end{array}\right),
\end{aligned}
$$

where

$$
\begin{aligned}
A_{1}= & \gamma_{S}-\omega_{S}-\mu_{S}-\frac{1}{\tau} \int_{0}^{\tau}\left(\sigma_{G} e^{z(t)}+\frac{\gamma_{S}}{K_{T}}\left(e^{x(t)}+e^{y(t)}\right)\right) d t \\
& +\frac{1}{\tau} \int_{0}^{\tau} \gamma_{N S} e^{-x(t)} e^{y(t)}\left(1-\frac{e^{x(t)}+e^{y(t)}}{K_{T}}\right) d t+\frac{1}{\tau} \ln \left(1-\eta_{S} w\left(e^{z(\tau)}\right)\right), \\
A_{2}= & -\mu_{N S}+\frac{1}{\tau} \int_{0}^{\tau} \omega_{S} e^{x(t)} e^{-y(t)} d t \\
A_{3}= & \gamma_{G}-\mu_{G}-\frac{1}{\tau} \int_{0}^{\tau} \frac{\gamma_{G}}{K_{G}} e^{z(t)} d t-\frac{1}{\tau} \int_{0}^{\tau} \sigma_{N S} e^{y(t)} d t+\frac{1}{\tau} \ln \left(1-\eta_{G}\right), \\
B_{1}= & \int_{0}^{t}\left(-\omega_{S}-\mu_{S}-\sigma_{G} e^{z(s)}+\left(\gamma_{S}+\gamma_{N S} e^{y(s)} e^{-x(s)}\right)\left(1-\frac{e^{x(s)}+e^{y(s)}}{K_{T}}\right)\right) d s, \\
B_{2}= & \int_{0}^{t}\left(-\mu_{N S}+\omega_{S} e^{x(s)} e^{-y(s)}\right) d s, \\
B_{3}= & \int_{0}^{t}\left(\gamma_{G}\left(1-\frac{e^{z(s)}}{K_{G}}\right)-\sigma_{N S} e^{y(s)}-\mu_{G}\right) d s,
\end{aligned}
$$




$$
\begin{aligned}
C_{1}= & \frac{t}{\tau}\left(\int_{0}^{\tau}\left(-\omega_{S}-\mu_{S}-\sigma_{G} e^{z(s)}+\left(\gamma_{S}+\gamma_{N S} e^{y(s)} e^{-x(s)}\right)\left(1-\frac{e^{x(s)}+e^{y(s)}}{K_{T}}\right)\right) d s\right. \\
& \left.+\ln \left(1-\eta_{S} w\left(e^{z(\tau)}\right)\right)\right), \\
C_{2}= & \frac{t}{\tau} \int_{0}^{\tau}\left(-\mu_{N S}+\omega_{S} e^{x(s)} e^{-y(s)}\right) d s, \\
C_{3}= & \frac{t}{\tau}\left(\int_{0}^{\tau}\left(\gamma_{G}\left(1-\frac{e^{z(s)}}{K_{G}}\right)-\sigma_{N S} e^{y(s)}-\mu_{G}\right) d s+\ln \left(1-\eta_{G}\right)\right), \\
D_{1}= & \ln \left(1-\eta_{S} w\left(e^{z(\tau)}\right)\right), \\
D_{2}= & 0, \\
D_{3}= & \ln \left(1-\eta_{G}\right) .
\end{aligned}
$$

Clearly, $Q N$ and $K_{P}(I-Q) N$ are continuous then for any open bounded set $\Omega \subset X$, $Q N(\bar{\Omega})$ is bounded. Furthermore, let $t_{1}, t_{2} \in[0, \tau], u(t)=(x, y, z)(t)$,

$$
f(t, u(t))=\left(\begin{array}{c}
-\omega_{S}-\mu_{S}-\sigma_{G} e^{z}+\left(\gamma_{S}+\gamma_{N S} e^{y} e^{-x}\right)\left(1-\frac{e^{x}+e^{y}}{K_{T}}\right) \\
-\mu_{N S}+\omega_{S} e^{x} e^{-y} \\
\gamma_{G}\left(1-\frac{e^{z}}{K_{G}}\right)-\sigma_{N S} e^{y}-\mu_{G}
\end{array}\right)
$$

and

$$
a=\left(\begin{array}{c}
\ln \left(1-\eta_{S} w\left(e^{z(\tau)}\right)\right) \\
0 \\
\ln \left(1-\eta_{G}\right)
\end{array}\right)
$$

We have

$$
\begin{aligned}
&\left|K_{P}(I-Q) N\left(u\left(t_{2}\right)\right)-K_{P}(I-Q) N\left(u\left(t_{1}\right)\right)\right| \\
&=\mid \int_{0}^{t_{2}} f(s, u(s)) d s-\int_{0}^{t_{1}} f(s, u(s)) d s-\frac{t_{2}}{\tau}\left\{\int_{0}^{\tau} f(s, u(s)) d s+a\right\} \\
&+\frac{t_{1}}{\tau}\left\{\int_{0}^{\tau} f(s, u(s)) d s+a\right\} \mid \\
&=\left|\int_{t_{1}}^{t_{2}} f(s, u(s)) d s-\frac{\left(t_{2}-t_{1}\right)}{\tau}\left\{\int_{0}^{\tau} f(s, u(s)) d s+a\right\}\right| \\
& \leq\left|t_{2}-t_{1}\right| \max _{t \in[0, \tau]}|f(t, u(t))|+\frac{\left|t_{2}-t_{1}\right|}{\tau}\left(\tau \max _{t \in[0, \tau]}|f(t, u(t))|+a\right) \\
& \leq\left|t_{2}-t_{1}\right|\left(2 \max _{t \in[0, \tau]}|f(t, u(t))|+\frac{a}{\tau}\right)
\end{aligned}
$$

and

$$
\begin{aligned}
\left|K_{P}(I-Q) N(u(t))\right| & \leq|a|+\tau \max _{t \in[0, \tau]}|f(t, u(t))|+|a|+\tau \max _{t \in[0, \tau]}|f(t, u(t))| \\
& \leq 2\left(|a|+\tau \max _{t \in[0, \tau]}|f(t, u(t))|\right) .
\end{aligned}
$$

Then using relations (64), (65) and the Arzela-Ascoli's theorem (Sonntag (1997) [42] Theorem 3.1, Page 314) we deduce that 
$K_{P}(I-Q) N(\bar{\Omega})$ is compact. Thus, $N$ is a $L$-compact mapping on $\bar{\Omega}$. The isomorphism $J$ of $\operatorname{Im} Q$ onto $\operatorname{Ker} L$ may be defined by

$$
J: \operatorname{Im} Q \rightarrow X, \quad\left(\left(\begin{array}{c}
u \\
v \\
w
\end{array}\right),\left(\begin{array}{l}
0 \\
0 \\
0
\end{array}\right)\right) \rightarrow\left(\begin{array}{c}
u \\
v \\
w
\end{array}\right) .
$$

Now we reach the position to search for an appropriate open, bounded subset $\Omega$ for the application of the continuation theorem, i.e we search $M_{0}$ such that every $\tau$-periodic solution of system (11) - (2) satisfied $|x(t)|+|y(t)|+|z(t)| \leq M_{0}$ with $0 \leq t \leq \tau$.

Corresponding to the operator equation $L x=\beta N x, \beta \in(0,1)$, we have

$$
\begin{cases}\dot{x}(t) & =\beta\left[-\omega_{S}-\mu_{S}-\sigma_{G} e^{z}+\left(\gamma_{S}+\gamma_{N S} e^{y} e^{-x}\right)\left(1-\frac{e^{x}+e^{y}}{K_{T}}\right)\right], \quad t \neq t_{n} . \\ \dot{y}(t) & =\beta\left[-\mu_{N S}+\omega_{S} e^{x} e^{-y}\right], \quad t_{n+1}=t_{n}+\tau \\ \dot{z}(t) & =\beta\left[\gamma_{G}\left(1-\frac{e^{z}}{K_{G}}\right)-\sigma_{N S} e^{y}-\mu_{G}\right] \\ x\left(t^{+}\right)-x(t) & =\beta \ln \left(1-\eta_{S} w\left(e^{z}\right)\right), \quad t=t_{n} . \\ y\left(t^{+}\right)-y(t) & =0, \quad n=0,1,2, \ldots \\ z\left(t^{+}\right)-z(t) & =\beta \ln \left(1-\eta_{G}\right) .\end{cases}
$$

Suppose that $(x(t), y(t), z(t)) \in X$ is an arbitrary solution of system (66) for a certain $\beta \in(0,1)$. Integrating on both sides of (66) over the interval $[0, \tau]$, we obtain

$$
\left\{\begin{aligned}
\int_{0}^{\tau}\left[-\frac{\gamma_{S}}{K_{T}}\left(e^{x}+e^{y}\right)+\gamma_{N S} e^{y} e^{-x}\left(1-\frac{e^{x}+e^{y}}{K_{T}}\right)-\sigma_{G} e^{z}\right] d t & =\left(\omega_{S}+\mu_{S}-\gamma_{S}\right) \tau \\
& -\ln \left(1-\eta_{S} w\left(e^{z(\tau)}\right)\right), \\
\int_{0}^{\tau} \omega_{S} e^{x} e^{-y} d t & =\mu_{N S} \tau, \\
\int_{0}^{\tau}\left[\frac{\gamma_{G}}{K_{G}} e^{z}+\sigma_{N S} e^{y}\right] d t & =\left(\gamma_{G}-\mu_{G}\right) \tau+\ln \left(1-\eta_{G}\right) .
\end{aligned}\right.
$$

Note that assumptions of Theorem 2 lead

$$
\left(\gamma_{G}-\mu_{G}\right) \tau+\ln \left(1-\eta_{G}\right)>0
$$

Since $X$ is a Banach space and $(x(t), y(t), z(t)) \in X$, there exist $\bar{\xi}, \underline{\xi}, \bar{\eta}, \underline{\eta}, \bar{\tau}$ and $\underline{\tau}$ such that

$$
\begin{array}{ll}
x(\bar{\xi})=\max _{0 \leq t \leq \tau} x(t), & x(\underline{\xi})=\min _{0 \leq t \leq \tau} x(t), \\
y(\bar{\eta})=\max _{0 \leq t \leq \tau} y(t), \quad y(\underline{\eta})=\min _{0 \leq t \leq \tau} y(t), \\
z(\bar{\tau})=\max _{0 \leq t \leq \tau} z(t), \quad z(\underline{\tau})=\min _{0 \leq t \leq \tau} z(t) .
\end{array}
$$

It follows from system (67) that

$$
\begin{aligned}
\int_{0}^{\tau}|\dot{x}(t)| d t & \leq\left(\omega_{S}+\mu_{S}\right) \tau+\int_{0}^{\tau}\left|-\sigma_{G} e^{z}+\left(\gamma_{S}+\gamma_{N S} e^{y} e^{-x}\right)\left(1-\frac{e^{x}+e^{y}}{K_{T}}\right)\right| d t \\
& \leq\left(\omega_{S}+\mu_{S}\right) \tau+\sigma_{G} \tau e^{z(\bar{\tau})}+\int_{0}^{\tau}\left(\gamma_{S}+\gamma_{N S} e^{y} e^{-x}\right) d t \\
& \leq\left(\omega_{S}+\mu_{S}\right) \tau+\sigma_{G} \tau e^{z(\bar{\tau})}+\int_{0}^{\tau}\left(\gamma_{S}+\gamma_{N S} e^{y}\right) d t \\
& \leq\left(\omega_{S}+\mu_{S}+\gamma_{S}\right) \tau+\sigma_{G} \tau e^{z(\bar{\tau})}+\gamma_{N S} \tau e^{y(\bar{\eta})},
\end{aligned}
$$




$$
\begin{aligned}
\int_{0}^{\tau}|\dot{y}(t)| d t & \leq \mu_{N S} \tau+\int_{0}^{\tau}\left|\omega_{S} e^{x} e^{-y}\right| d t \\
& \leq 2 \mu_{N S} \tau
\end{aligned}
$$

and

$$
\begin{aligned}
\int_{0}^{\tau}|\dot{z}(t)| d t & \leq\left(\gamma_{G}+\mu_{G}\right) \tau+\int_{0}^{\tau}\left(\frac{\gamma_{G}}{K_{G}} e^{z}+\sigma_{N S} e^{y}\right) d t \\
& \leq\left(\gamma_{G}+\mu_{G}\right) \tau+\left(\gamma_{G}-\mu_{G}\right) \tau+\ln \left(1-\eta_{G}\right) \\
& \leq 2 \gamma_{G} \tau+\ln \left(1-\eta_{G}\right) .
\end{aligned}
$$

Recall that

$$
\begin{aligned}
\rho_{G}^{0}>1 & \Longleftrightarrow\left(\gamma_{G}-\mu_{G}\right) \tau+\ln \left(1-\eta_{G}\right)>0 \\
& \Longrightarrow 2 \gamma_{G} \tau+\ln \left(1-\eta_{G}\right)>0 .
\end{aligned}
$$

Since

$$
\begin{aligned}
\tau\left(\frac{\gamma_{G}}{K_{G}} e^{z(\underline{\tau})}+\sigma_{N S} e^{y(\underline{\eta})}\right) & \leq\left(\gamma_{G}-\mu_{G}\right) \tau-\int_{0}^{\tau} \dot{z}(t) d t \\
& \leq\left(\gamma_{G}-\mu_{G}\right) \tau+\ln \left(1-\eta_{G}\right),
\end{aligned}
$$

then

$$
\begin{aligned}
& z(\underline{\tau}) \leq \ln \left\{\frac{K_{G}}{\gamma_{G}}\left(\left(\gamma_{G}-\mu_{G}\right)+\frac{\ln \left(1-\eta_{G}\right)}{\tau}\right)\right\} \\
& y(\underline{\eta}) \leq \ln \left\{\frac{1}{\sigma_{N S}}\left(\left(\gamma_{G}-\mu_{G}\right)+\frac{\ln \left(1-\eta_{G}\right)}{\tau}\right)\right\} .
\end{aligned}
$$

Moreover, from

$$
\int_{0}^{\tau} \omega_{S} e^{x} e^{-y} d t=\mu_{N S} \tau
$$

we deduce

$$
x(\underline{\xi}) \leq \ln \left(\frac{\mu_{N S}}{\omega_{S}} e^{y(\bar{\eta})}\right) \text { and } x(\bar{\xi}) \geq \ln \left(\frac{\mu_{N S}}{\omega_{S}}\right) .
$$

Furthermore,

$$
\int_{0}^{\tau} \dot{z}(t) d t=\int_{0}^{\tau}\left[\gamma_{G}-\mu_{G}-\frac{\gamma_{G}}{K_{G}} e^{z(t)}-\sigma_{N S} e^{y(t)}\right]=-\ln \left(1-\eta_{G}\right) .
$$

Using

$$
\begin{aligned}
0<\left(\gamma_{G}-\mu_{G}\right) \tau+\ln \left(1-\eta_{G}\right) & =\int_{0}^{\tau}\left[\frac{\gamma_{G}}{K_{G}} e^{z(t)}+\sigma_{N S} e^{y(t)}\right] d t \\
& \leq \int_{0}^{\tau}\left[\frac{\gamma_{G}}{K_{G}} e^{z(\bar{\tau})}+\sigma_{N S} e^{y(\bar{\eta})}\right] d t=\tau\left[\frac{\gamma_{G}}{K_{G}} e^{z(\bar{\tau})}+\sigma_{N S} e^{y(\bar{\eta})}\right]
\end{aligned}
$$

then there exist $\zeta_{1}>0, \zeta_{2}>0$ such that

- $\zeta_{1}+\zeta_{2}=\left(\gamma_{G}-\mu_{G}\right)+\frac{\ln \left(1-\eta_{G}\right)}{\tau}$,

- $\frac{\gamma_{G}}{K_{G}} e^{z(\bar{\tau})} \geq \zeta_{1}$ and 
- $\sigma_{N S} e^{y(\bar{\eta})} \geq \zeta_{2}$.

Thus,

$$
z(\bar{\tau}) \geq \ln \left\{\frac{K_{G}}{\gamma_{G}} \zeta_{1}\right\}, y(\bar{\eta}) \geq \ln \left\{\frac{1}{\sigma_{N S}} \zeta_{2}\right\} .
$$

So, keeping in mind that by assumptions of Theorem 2, one has

$$
\left(\gamma_{G}-\mu_{G}\right)+\frac{\ln \left(1-\eta_{G}\right)}{\tau}>0
$$

$$
\begin{aligned}
x(t) & \leq x(\underline{\xi})+\int_{0}^{\tau}|\dot{x}(t)| d t & \\
& \leq \ln \left(\frac{\mu_{N S}}{\omega_{S}} e^{y(\bar{\eta})}\right)+\left(\omega_{S}+\mu_{S}+\gamma_{S}\right) \tau+\sigma_{G} \tau e^{z(\bar{\tau})}+\gamma_{N S} \tau e^{y(\bar{\eta})} & :=x_{u}, \\
y(t) & \leq y(\underline{\eta})+\int_{0}^{\tau}|\dot{y}(t)| d t & \\
& \leq \ln \left\{\frac{1}{\sigma_{N S}}\left(\left(\gamma_{G}-\mu_{G}\right)+\frac{\ln \left(1-\eta_{G}\right)}{\tau}\right)\right\}+2 \mu_{N S} \tau & :=y_{u}, \\
z(t) & \leq z(\underline{\tau})+\int_{0}^{\tau}|\dot{z}(t)| d t & \\
& \leq \ln \left\{\frac{K_{G}}{\gamma_{G}}\left(\left(\gamma_{G}-\mu_{G}\right)+\frac{\ln \left(1-\eta_{G}\right)}{\tau}\right)\right\}+2 \gamma_{G} \tau+\ln \left(1-\eta_{G}\right) & :=z_{u}, \\
x(t) & \geq x(\bar{\xi})-\int_{0}^{\tau}|\dot{x}(t)| d t & :=x_{l}, \\
& \geq \ln \left(\frac{\mu_{N S}}{\omega_{S}}\right)-\left(\omega_{S}+\mu_{S}+\gamma_{S}\right) \tau-\sigma_{G} \tau e^{z(\bar{\tau})}-\gamma_{N S} \tau e^{y(\bar{\eta})} & \\
y(t) & \geq y(\bar{\eta})-\int_{0}^{\tau}|\dot{y}(t)| d t & \\
& \geq \ln \left\{\frac{\zeta_{2}}{\sigma_{N S}}\right\}-2 \mu_{N S} \tau & :=y_{l}, \\
z(t) & \geq z(\bar{\tau})-\int_{0}^{\tau}|\dot{z}(t)| d t & \\
& \geq \ln \left\{\frac{K_{G}}{\gamma_{G}} \zeta_{1}\right\}-2 \gamma_{G} \tau-\ln \left(1-\eta_{G}\right) &
\end{aligned}
$$

therefore we obtain,

$$
\begin{aligned}
& \max _{0 \leq t \leq \tau}|x(t)| \leq \max \left\{\left|x_{u}\right|,\left|x_{l}\right|\right\}:=M_{x} \\
& \max _{0 \leq t \leq \tau}|y(t)| \leq \max \left\{\left|y_{u}\right|,\left|y_{l}\right|\right\}:=M_{y} \\
& \max _{0 \leq t \leq \tau}|z(t)| \leq \max \left\{\left|z_{u}\right|,\left|z_{l}\right|\right\}:=M_{z} .
\end{aligned}
$$

$M_{x}, M_{y}$ and $M_{z}$ are independent of $\beta$.

Now let us consider the algebraic equations

$$
\left\{\begin{aligned}
\gamma_{S}-\omega_{S}-\mu_{S}+\frac{1}{\tau} \ln \left(1-\eta_{S} w\left(e^{z(\tau)}\right)\right) & \\
-\frac{1}{\tau} \int_{0}^{\tau}\left[\frac{\gamma_{S}}{K_{T}}\left(e^{x}+e^{y}\right)-\beta \gamma_{N S} e^{y} e^{-x}\left(1-\frac{e^{x}+e^{y}}{K_{T}}\right)+\beta \sigma_{G} e^{z}\right] d t & =0 \\
-\mu_{N S}+\frac{1}{\tau} \int_{0}^{\tau} \omega_{S} e^{x} e^{-y} d t & =0 \\
\left(\gamma_{G}-\mu_{G}\right)+\frac{1}{\tau} \ln \left(1-\eta_{G}\right)-\frac{1}{\tau} \int_{0}^{\tau}\left[\frac{\gamma_{G}}{K_{G}} e^{z}+\sigma_{N S} e^{y}\right] d t & =0
\end{aligned}\right.
$$

for $(x, y, z) \in \mathbb{R}^{3}$, where $\beta \in[0,1]$ is a parameter. By carrying out similar arguments as in system (67), one can show that any solution $\left(x^{*}, y^{*}, z^{*}\right)$ of (178) with $\beta \in[0,1]$ satisfies

$$
l_{1} \leq x^{*} \leq L_{1}, \quad l_{2} \leq y^{*} \leq L_{2}, \quad l_{3} \leq z^{*} \leq L_{3} .
$$


Taking $M_{0}=M_{x}+M_{y}+M_{z}+M_{k}$ where $M_{k} \dot{\iota} 0$ is taken sufficiently large such that $M_{k}>\left|l_{1}\right|+\left|L_{1}\right|+\left|l_{2}\right|+\left|L_{2}\right|+\left|l_{3}\right|+\left|L_{3}\right|$, we define $\Omega=\left\{(x, y, z)^{T} \in X:\|(x, y, z)\|<M_{0}\right\}$, then $\Omega$ verifies the requirement (1) of The Continuation Theorem (Gaines and Mahwin (1977) [27], Page 40). When $(x, y, z) \in \partial \Omega \cap \operatorname{Ker} L=\partial \Omega \cap \mathbb{R}^{3},(x, y, z)$ is a constant vector in $\mathbb{R}^{3}$ with $\|(x, y, z)\|=M_{0}$. Then from (79) and the definition of $M_{0}$, one has

$$
Q N\left(\begin{array}{l}
x \\
y \\
z
\end{array}\right)=\left(\left(\begin{array}{l}
A^{(1)} \\
A^{(2)} \\
A^{(3)}
\end{array}\right),\left(\begin{array}{l}
0 \\
0 \\
0
\end{array}\right)\right) \neq\left(\left(\begin{array}{l}
0 \\
0 \\
0
\end{array}\right),\left(\begin{array}{l}
0 \\
0 \\
0
\end{array}\right)\right),
$$

where,

$$
\begin{aligned}
A^{(1)}= & \gamma_{S}-\omega_{S}-\mu_{S}-\frac{1}{\tau} \int_{0}^{\tau}\left(\sigma_{G} e^{z}+\frac{\gamma_{S}}{K_{T}}\left(e^{x}+e^{y}\right)\right) d t+\frac{1}{\tau} \int_{0}^{\tau} \gamma_{N S} e^{-x} e^{y}\left(1-\frac{e^{x}+e^{y}}{K_{T}}\right) d t \\
& +\frac{1}{\tau} \ln \left(1-\eta_{S} w\left(e^{z(\tau)}\right)\right), \\
A^{(2)=} & -\mu_{N S}+\frac{1}{\tau} \int_{0}^{\tau} \omega_{S} e^{x} e^{-y} d t, \\
A^{(3)=} & \gamma_{G}-\mu_{G}-\frac{1}{\tau} \int_{0}^{\tau} \frac{\gamma_{G}}{K_{G}} e^{z} d t-\frac{1}{\tau} \int_{0}^{\tau} \sigma_{N S} e^{y} d t+\frac{1}{\tau} \ln \left(1-\eta_{G}\right),
\end{aligned}
$$

that is, the first part of (2) of The Continuation Theorem (Gaines and Mahwin (1977) [27], Page 40) is valid.

To compute the Brouwer degree, let us consider the homotopy

$$
H_{\beta}\left((x, y, z)^{T}\right)=\beta J Q N\left((x, y, z)^{T}\right)+(1-\beta) V\left((x, y, z)^{T}\right), \quad \beta \in[0,1],
$$

where

$$
V\left((x, y, z)^{T}\right)=\left(\begin{array}{c}
\gamma_{S}-\omega_{S}-\mu_{S}+\frac{1}{\tau} \ln \left(1-\eta_{S} w\left(e^{z(\tau)}\right)\right)-\frac{\gamma_{S}}{K_{T}}\left(e^{x}+e^{y}\right) \\
-\mu_{N S}+\omega_{S} e^{x} e^{-y} \\
\left(\gamma_{G}-\mu_{G}\right)+\frac{1}{\tau} \ln \left(1-\eta_{G}\right)-\frac{\gamma_{G}}{K_{G}} e^{z}-\sigma_{N S} e^{y}
\end{array}\right)
$$

From (78), it follows that $0 \notin H_{\beta}(\partial \Omega \cap K e r L)$ for $\beta \in[0,1]$. Moreover, since $-\frac{\gamma_{S} \gamma_{G}\left(\omega_{S}+\mu_{N S}\right)}{K_{T} K_{G}} \neq$ 0 , the algebraic equation $V\left((x, y, z)^{T}\right)=0$ has a unique solution $\left(e^{x^{*}}, e^{y^{*}}, e^{z^{*}}\right)^{T} \in \mathbb{R}^{3}$. We compute the Brouwer degree $(\operatorname{deg}(\cdot, \cdot, \cdot))$ by using the invariance property of homotopy [?], one has

$$
\begin{aligned}
& \operatorname{deg}(J Q N, \Omega \cap \operatorname{Ker} L, 0)=\operatorname{deg}(V, \Omega \cap \operatorname{Ker} L, 0) \\
& =\sum_{p \in V^{-1}(0)} \operatorname{sign}\left(J_{V}(p)\right) \\
& \operatorname{sign}\left[\operatorname{det}\left(\begin{array}{ccc}
-\frac{\gamma_{S}}{K_{T}} e^{x^{*}} & -\frac{\gamma_{S}}{K_{T}} e^{y^{*}} & 0 \\
\mu_{N S} & -\mu_{N S} & 0 \\
0 & -\sigma_{N S} e^{y^{*}} & -\frac{\gamma_{G}}{K_{G}} e^{z^{*}}
\end{array}\right)\right] \\
& =\operatorname{sign}\left[-\frac{\gamma_{G} \gamma_{S}}{K_{T} K_{G}}\left(\mu_{N S}+\omega_{S}\right) e^{x^{*}} e^{z^{*}}\right] \text {, since } \omega_{S} e^{x^{*}}=\mu_{N S} e^{y^{*}} \\
& =-1 \neq 0 \text {. }
\end{aligned}
$$


By now, we have prove that $\Omega$ verifies all requirements of The Continuation Theorem (Gaines and Mahwin (1977) [27], Page 40), then $L x=N x$ has at least one solution in DomL $\cap \bar{\Omega}$, i.e. system (57) has at least one solution in $\operatorname{Dom} L \cap \bar{\Omega}$, say $\left(x^{*}(t), y^{*}(t), z^{*}(t)\right)^{T}$. Set $T_{S}^{*}(t)=$ $\exp \left(x^{*}(t)\right), T_{N S}^{*}(t)=\exp \left(y^{*}(t), G^{*}(t)=\exp \left(z^{*}(t)\right)\right.$, then $\left(T_{S}^{*}(t), T_{N S}^{*}(t), G^{*}(t)\right)^{T}$ is a positive and $\tau$-periodic solution of system (11) - (2). This completes the proof.

\section{Appendix D: Particular values of $\sigma_{N S}, \sigma_{G}$ and $\tau$}

With respect to relations (13) and (17), we set

$$
\left\{\begin{aligned}
\sigma_{G}^{\star}(\tau) & =\frac{1}{G_{i n t}}\left(\gamma_{S}-\left(\mu_{S}+\omega_{S}+\mu_{N S}\right)\right), \\
& =\frac{\left(\gamma_{S}-\left(\mu_{S}+\omega_{S}+\mu_{N S}\right)\right)}{\frac{K_{G}}{\gamma_{G}}\left(\gamma_{G}-\mu_{G}+\frac{\ln \left(1-\eta_{G}\right)}{\tau}\right)}, \\
\sigma_{N S}^{\star}(\tau) & =\frac{1}{\bar{T}_{N S}}\left(\gamma_{G}-\mu_{G}+\frac{\ln \left(1-\eta_{G}\right)}{\tau}\right), \\
\tau^{\star} & =-\frac{\ln \left(1-\eta_{G}\right)}{\gamma_{G}\left(1-\frac{1}{\mathcal{R}_{T}^{G}}\right)} .
\end{aligned}\right.
$$

One can note that $\sigma_{G}^{\star}, \sigma_{N S}^{\star}$ and $\tau^{\star}$ determined regions of stability/instability of forest and grassland solutions, with respect to $\sigma_{G}, \sigma_{N S}$ and $\tau$ variations. 